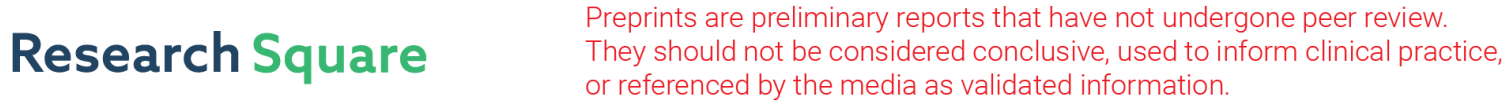 \\ Process Monitoring in a Flexible Manufacturing Cell through Data Analytics and a Visualisation Tool
}

Giovanna Martinez Arellano ( $\sim$ giovanna.martinezarellano@nottingham.ac.uk)

University of Nottingham

\section{ThuBa Nguyen}

Cranfield University

Chris Hinton

BMW Group

\section{Svetan Ratchev}

University of Nottingham

\section{Research Article}

Keywords: flexible manufacturing systems, statistical process control, data analytics, data visualisation

Posted Date: April 7th, 2021

DOl: https://doi.org/10.21203/rs.3.rs-299328/v1

License: (c) (1) This work is licensed under a Creative Commons Attribution 4.0 International License.

Read Full License 


\title{
Process Monitoring in a Flexible Manufacturing Cell through Data Analytics and a Visualisation Tool
}

\author{
Giovanna Martínez-Arellano - ThuBa Nguyen • Chris Hinton • Svetan \\ Ratchev
}

Received: date / Accepted: date

\begin{abstract}
With the need of more responsive and resilient manufacturing processes for high value, customised products, Flexible Manufacturing Systems (FMS) remain a very relevant manufacturing approach. Due to their complexity, quality monitoring in these types of systems can be very difficult, particularly in those scenarios where the monitoring cannot be fully automated due to functional, safety and legal characteristics. In these scenarios, quality practitioners concentrate on monitoring the most critical processes and leaving out the inspection of those that are still meeting quality requirements but showing signs of future failure. In this paper we introduce a methodology and visualisation tool based on data analytics that allows the practitioner to anticipate out of control processes and take action. By identifying a reference model or best performing machine, and the occurring patterns in the quality data, the presented approach identifies the adjustable processes that are still in control, allowing the practitioner to decide if any changes in the machine's settings are needed (tool replacement, repositioning the axis, etc). An initial deployment of the tool at BMW Plant Hams Hall to monitor a focussed set of part types and features has shown a reduction in scrap of $97 \%$ throughout 2020
\end{abstract}

Giovanna Martínez-Arellano and Svetan Ratchev

Institute for Advanced Manufacturing, Jubilee Campus,

University of Nottingham, Nottingham NG7 2GX, UK

E-mail: giovanna.martinezarellano@nottingham.ac.uk

E-mail: svetan.ratchev@nottingham.ac.uk

ThuBa Nguyen

School of Aerospace, Transport and Manufacturing, Cranfield

University

E-mail: ThuBa.Nguyen@cranfield.ac.uk

Chris Hinton

Hams Hall Plant, BMW Group, Birmingham, UK

E-mail: chris.hinton@bmwgroup.com in relation to the monitored features compared to the previous year. This in the long run will reduce reaction time in following quality control procedure, reduce significant scrap costs and ultimately reduce the need for measurements and enable more output in terms of volume capacity.

Keywords flexible manufacturing systems · statistical process control $\cdot$ data analytics $\cdot$ data visualisation

\section{Introduction}

Flexibility in manufacturing systems is becoming more and more necessary as domestic and global markets rapidly change, technology evolves and demand towards more customisable products increases. Flexibility in manufacturing allows companies to be able to deal with fast changing product types, production volumes, assembly variation and process sequence while keeping production processes time and cost effective [2, 51. Flexible manufacturing systems (FMS) achieve these goals with programmable automation through the use of a number of systems such as CNC machine tools, interconnected by automated material handling and storage systems, inspection stations and gauging systems 37.

The reliability of the automated equipment in FMS is a critical contributor to system performance, and so the capability of performing correct and rapid fault diagnosis and process variation identification through Statistical Process Control (SPC) is essential [8]. SPC and the use of control charts can detect process variations and identify possible machine breakdowns and out-of-control processes that can affect the availability of resources. However, it has been found that traditional SPC approaches are usually not appropriate for production paradigms such as flexible and re-configurable 
systems, where the production is small or there is a high variety of mixed products. Short production runs pose several challenges. First, the lack of available data to estimate reliable process parameters due to smaller production runs. This is a typical scenario in Just-intime (JIT) systems, where low levels of inventory are kept, and during start-up of a process or initiation of a new process, where there is an insufficient number of subgroups of measurements under different conditions available 38. Second, there is an increasing risk of false acceptance in SPC because of measurement errors [10]. Several possible sources of error are equipment accuracy, operator mistakes, environmental factors and random noise. Measurement errors can lead to unnecessary process adjustments and loss of confidence in SPC 32. Third, the production of customised products and services are characterised by complex designs and processes, requiring more flexible quality management practices. SPC in flexible environments requires more control charts to monitor quality characteristics and product specifications compared to more static approaches 33. The data generated by analysing and monitoring individual process, product family and test stations can be extemently large and hard to manage [21]. Manufacturing companies still rely on operator and quality practitioner experience in analysing the information. There are product characteristics within manufacturing which need particular attention because of their functional, safety and legal characteristics requirements. These types of features often carry tight tolerances or parameters which are particularly sensitive to variation and change in the production systems. This is why employees who are involved in the collection and analysis of the data need to understand statistical concepts and how they can be interpreted to process control 26].

In a flexible manufacturing system, where the number of machines, component variants and product characteristics to control is so high, the operator will prioritise the analysis and reaction method of those processes which tolerance limits are breached, disregarding other processes that might not be running at the expected standards. Despite the development of new SPC methods to deal with the challenges of FMS (e.g. change-point methods, pattern recognition using Machine Learning), these normally solve some but not all the issues [15, 33, 38, and are often very difficult to implement by the non-expert. In addition, there is little literature related to the implementation of these new SPC methods in industry.

This paper presents the implementation of a methodology and data visualisation tool for process monitoring in a real industrial scenario. The proposed method- ology uses classical SPC together with advanced data analytics algorithms to automate the analysis of quality data in near real-time, allowing the practitioner to anticipate problems before processes go out of control. By automatically detecting patterns, the visualisation tool supports quality practitioners in indicating which machines need adjusting based on a reference model or lead machine. This reference model is determined automatically by considering the most recent available data. The tool is used and evaluated in a flexible manufacturing environment at BMW, where the number of machines, component variants and features per component are high, demonstrating the benefit of the tool compared to the typical monitoring methods used in such environment. The rest of the paper is organised as follows: Section 2 presents work related to recent developments of SPC techniques particularly for FMS. The methodology is then presented in Section 3, followed by a detailed description of the case study at BMW and a discussion of results in Section 4. Finally, Section 5 presents the conclusions and future work.

\section{Related Work}

Although flexible manufacturing is not a new paradigm, there has been growing interest in recent years due to factors including the growing demand for customised products, shorter product life cycles and environmental impact [23, 29, 60]. Compared to traditional transfer lines that enable high volume but less flexibility in their design, FMS allow for reaction to volume fluctuations, mixed batch production and multiple product types within the same product family [9]. This flexibility involves having machine capabilities that are themselves flexible (such as CNC machines) and hence can produce multiple product variants, but at the cost of making production lines more complex and difficult to monitor. Like in any manufacturing paradigm, SPC plays an important part in FMS to ensure that manufacturing processes operate in their in-control state. However, it has been found that traditional SPC methods are not appropriate for situations of small lots or where a high variety of products exist [38. Although flexible production systems may manufacture large volumes, the production in these types of environments is intermittent because the change to other product variants is easy. This intermittent aspect makes traditional SPC methods particularly unsuitable. In addition, whilst being highly automated, the balance required between quality control interruption and intelligence driven solutions needs to be addressed. A typical process within a production line in an FMS would require the inspection of tens of thousands of control charts, making it possible 
for the operator to miss processes or features which have been affected or shifted but still fall within acceptable limits.

\subsection{SPC Control Charts}

Traditional control charts such as Shewhart, Cumulative Sum (CUSUM) and Exponentially Weighted Moving Average (EWMA) assume that the values of a Process Mean and Variance are known a priori at the start of the process monitoring or that the data for estimating the process parameters is available during a production run [41. Traditional SPC is based on the assumption that the data is Independent and Identically Normally Distributed (IIND). However, in environments such as an FMS where there is a variety of mixed products processed in the same production line, these fundamental assumptions are not met. As different measured part features may have different means and variances, the IIND condition is not satisfied.

There are many methods that have been developed to address the limitations of classical SPC methods in FMS. Hillier developed methods to adjust control limits used in X-bar and $\mathrm{R}$ control charts [24. Quesenberry proposed $\mathrm{Q}$ charts, which can detect changes in the mean and variance in short production runs [44. These charts have been further studied and enhanced in other works [6, 20, 59, as it has been found that the method is sensitive to early shifts of the mean, or when the variance is unknown. T-charts are other new type of control charts based on the calculation of the T-statistic and were first proposed by Zhang et al [34]. A T-chart does not require a preliminary estimation of the in-control process Standard Deviation (SD), making it useful when monitoring at the startup of a process. However, T-charts rely on setting up the control mean at the beginning of the production run. Further developments of T-charts have been done by [4, 5, 15]. Many other approaches have dealt with the normality and non independent assumption validations as well as the small sample size such the ARMA chart [27], ML-chart [53], ACUSUM charts [54.

\subsection{Change-point SPC}

In general, control charts allow to determine if a process is in or out of control. However, in some circumstances, by the time the process has reached the control limit and an alert has been raised, it might be already too late to avoid or fix the related issue. An early detection of a trend or pattern in the measurements before reaching the control limit might allow the operator to identify and react on time to a possible future issue. To complement charts, methods to detect shifts in shortrun situations such as the change-point method have been proposed [1]. The basic change-point approach, as introduced by Hawkins, is based on the assumption that process readings can be modeled by two normal distributions. Process readings follow an initial distribution until certain point $\tau$, the change-point, at which point they switch to another normal distribution, differing in the mean, variance or both [19]. Methods to monitor the process mean and process variance in univariate and multivariate applications using the change-point method have been proposed in [43, 57. In short-run environments where data is scarce, outliers can have a big impact on detecting true change-points. Methods with particular focus on outliers have also been proposed [13, 55. To deal with data that is not normally distributed, non-parametric solutions have been proposed as well [18, 61]. Although a considerable amount of work has been done on these methods, there is still work to be done, particularly in non-parametric techniques [28]. Jones-Farmer et al. particularly stress that for more quality-control practitioners to use these methods on real scenarios, there needs to be more development of easy-to-use computer software that can make these complex methods accessible to them [28].

\subsection{Control chart pattern recognition}

In addition to change-point based strategies, there are other methods based on Machine Learning (ML) that have been applied for detecting patterns in process quality data. Different detected patterns may be an indication of different problems. While trend patterns may indicate a slow change, cyclic patterns might be related to periodic variation in the process such as power supply [58. In the early application of control charts, it was necessary to manually determine whether or not there was an abnormality, this being more difficult when the process was still in control. To this, many pattern detection methods based on supplementary rules where proposed [30, 52. Wang et al. defined 30 possible control chart patterns, categorised as 8 basic patterns and 22 combinations of the basic ones 52 . However, dealing with a large number of rules may be difficult when doing real-time monitoring. To address this, recent work in applying ML for pattern recognition has been done with the aim to automate this process [7, 50, 58, 62].

Control chart pattern recognition methods have been incorporated with other SPC methods in an attempt to provide more complete process monitoring tools (i.e. online monitoring, diagnosis and prescription) that could support the quality practitioner to take action in real 
time. Guh developed an on-line SPC system that incorporates an Artificial Neural Network (ANN) to perform pattern recognition together with an expert system that can interpret or relate the patterns recognised with possible issues and propose solutions to the quality practitioner [17. Other online process monitoring and diagnosis approaches that combine ML techniques have been reported [22, 56].

Root cause analysis and diagnosis has been a main focus of the research in process monitoring in recent years. However, there is another dimension to process monitoring that is still to be reached: Prognosis i.e. determining the likely development and change of a problem. Current trends in industrial process monitoring are moving towards this direction [12, 45]. Despite the ampunt of work published in ML-based monitoring and diagnostic methods, most of these are tested in a simulated scenario, there is very little reported on industrial case studies for these tools. In addition, more flexibility is still needed so that these types of approaches can be used by practitioners. Most ML applications are still inaccessible to most practitioners due to their complexity and required sills needed for their implementation and continued use.

\subsection{Computerised tools in real industry scenarios}

Along with the development of new methods for improving process control, there have been efforts for implementing these in real scenarios which involve the development of computational tools for SPC implementation and data visualisation. Azadeh and Zeynali developed a framework of integrated quality control that features a Quality Information System, Statistical Quality Control, and SPC. The system was tested for a 5-year period in a large industrial machinery manufacturer, demonstrating the benefits of the computerised system [3. Huang et al performed a case study in a Taiwanese LCD manufacturer where they study the key factors that can make an SPC system successful by developing an effective performance evaluation model. The authors claim that despite the success of large automotive companies implementing the SPC manual in QS-9000 (quality stanard which has been around since 1994), most organisations are still learning about the most effective ways to introduce, develop and implement SPC. One of the key factors that influenced the success of the implementation of SPC in a business was adequate training and support for the users of the system through either internal education or external experts and advisors [25]. Guerra et al. developed an SPC software tool that was used to automate the quality control in the final inspection process of a production line in an automotive company. The authors stressed the lack to published work related to case studies in industry and how industry is dealing with the implementation of computerised SPC systems [16.

\subsection{Big Data Analytics and Visualisation for Industry} 4.0

With the advances in Information Technology (IT) and increased computing power, data analytics at both small and large scales are now possible. There is a vast amount of work done in the development and implementation of advanced data analytics methods for condition monitoring, fault diagnosis, among other process industry applications [14, 42, 46, 47, 48, 49]; and these developments are often considered part of Industry 4.0. Data analytics dashboards can now be easily developed through tools such as PowerBI or open source technologies such as R and Grafana. However, despite the vast amount of research on Industry 4.0 architecture technologies and applications, industries are still not confident enough on the implementation of these technologies due to the unclear benefits as well as the lack of implementation details and the investment needed 36. The challenges that still remain in industry are:

- Process complexity - monitoring a large amount of process parameters/quality features to identify issues as they rely heavily on the quality practitioner's expertise.

- Data size - Large data volumes in terms of number of process variations/parameters but lack of data/measurements of one specific process variation in a FMS environment.

- Technical expertise - there is still scepticism from industry in regards to ML applications, and a lack of practitioners with the necessary skills.

This work attempts to address these challenges through the development and implementation of a process monitoring methodology and visualisation tool. The methodology allows to identify normal and out-of-control process behaviour and goes a bit further by allowing the practitioner to anticipate potential issues.

\section{Methodology}

By exploiting the current advances in data analytics, this work presents a methodology that incorporates classic and advanced SPC methods together with data analytics algorithms to perform the identification of potential problems in processes before these reach the specified control limits. This is performed by identifying, -in 


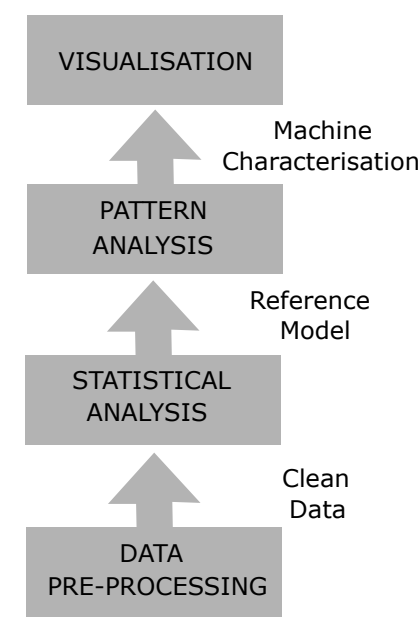

Fig. 1 Methodology used to automatically identify incontrol, out-of-control and adjustable processes in a flexible manufacturing line.

an online manner-, the best performing process (e.g. machine) with as few as 5 measurements and using this to identify other processes that can be potentially improved as their performance moves away from the best case. As it is shown in Figure 1, there are four major steps performed in the aforementioned methodology: data pre-processing, reference model identification, machine characterisation and visualisation. These are described in more detail in the following subsections.

\subsection{Data pre-processing}

FMS are characterised for the complexity and variation of its processes. The methodology described in this work is intended for FMS environments, where the number of process variations and features within one process variation is large. Particularly, this work is applied in an automotive industrial environment with the following characteristics:

- There is a number of component variations (i.e. part types) depending on demand

- Each part type has hundreds of product characteristics (i.e. features) to monitor across multiple machines.

- One machine is capable of producing the same features of multiple components variants to achieve high volume production.

These system factors determine the data complexity. The number of features (e.g. geometrical features measured with a Coordinate Measuring Machine CMM) for each part type variant can be very large, but at the same time, the number of continuous measurements for each machine for a particular feature/part variant can be small. Before performing any statistical analysis related to the performance of a machine using product features, the data is pre-processed by eliminating any duplicate measurements and outliers that can be related to errors in the CMM measurements. As mentioned previously, outliers can have a large effect on the estimation of process control statistics, particularly when data is scarce. Most common outlier detection methods are based on the Standard Deviation (SD) over the mean. However, this is fundamentally problematic, as the indicator itself is altered by the presence of outliers. The absolute deviation from the median method (MAD), on the other hand, while still being a measure of central tendency, is insensitive to the presence of outliers and immune to sample size, making it a better indicator 35. Calculating the MAD involves finding the median of absolute deviations from the median. It is defined as follows:

$M A D=b M_{i}\left(\left|x_{i}-M_{j}\left(x_{j}\right)\right|\right)$

where the $x_{j}$ is the $n$ original observations, $M_{i}$ is the median of the series and $\mathrm{b}$ is a constant, usually $b=$ 1.4826, linked to the assumption of normality of the data. The constant $\mathrm{b}$ ensures that for large samples, the MAD remains a consistent estimator of the SD of the population. The decision criteria is then defined as:

$M-t * M A D<x_{i}<M+t * M A D$

where $t$ is the threshold selected 40]. In this methodology, MAD is applied on all quality measurements per machine and per feature, and across multiple part types on a given time frame. An example of outliers detected for a feature $F$ in a set of machines can be seen in Figure 2 .

3.2 Statistical Analysis to determine Reference Model and Adjustable Machines

Once the data is cleaned, the first step to identify the best performing machine in a particular period of time (determined by the user but at least 5 measurements required) is to calculate for each feature measurement its deviation from the nominal or target value, this could be the real deviation or the percentage. The deviation of a measurement $m$ is calculated as follows:

First, the nominal value related to each measurement is determined. This value is zero in the case of unilateral tolerances, otherwise the nominal value is:

nominalValue $=u C L-l C L$ 


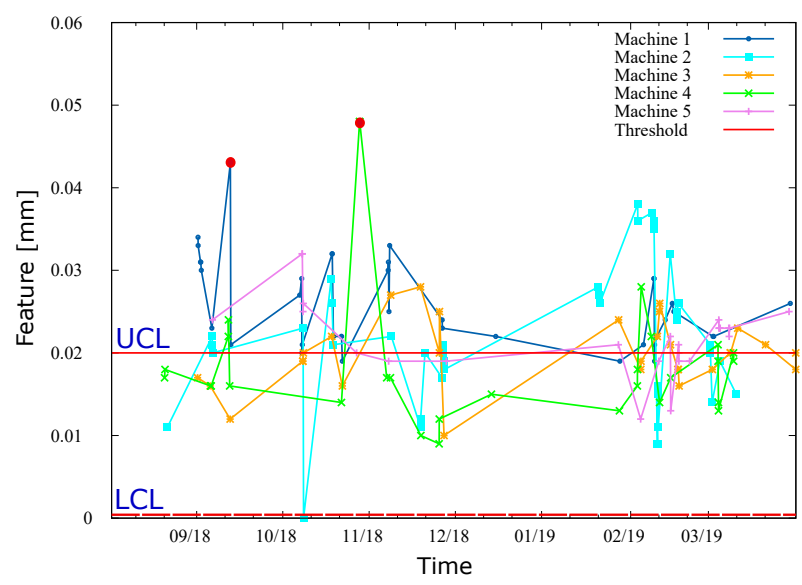

Fig. 2 Outliers (red dots) identified on CMM measurements of a particular feature $F$ corresponding to 5 machines. Although the algorithm picks up an outlier in machine A_-7_1, this measurement is not considered an outlier in this particular case as the target value for this feature is zero.

where uCL stands for upper control limit and lCL for lower control limit. From this nominal value, then a deviation from target can be calculated:

targetDeviation $_{m}=\mid$ nominalValue $-m \mid$

For the percentage deviation, if it is the case of an unilateral tolerance then:

percentage Deviation $_{m}=\frac{m}{u C L} \times 100$

If the lower control limit of the feature is different from zero:

percentageDeviation $_{m}=\frac{\text { targetDeviation }_{m}}{\text { nominalValue } \times \frac{1}{2}} \times 100$

The percentage deviation is useful when using multiple features for the best machine calculation when these might have different tolerances. In a second step, the target deviation is used to calculate a set of statistical features per feature and machine. These include:

- Mean, SD and Variance of the feature measurement $m$.

- Minimum and maximum measurement.

- Mean and SD of the deviation of the measurement from the target value.

- Minimum and maximum deviation from target.

- Number of measurements out of threshold

These statistical features will be used for the best machine estimation as well as for constructing the visualisation dashboard which provides not only an estimated best machine but also statistical information on other machines that could be performing similarly.

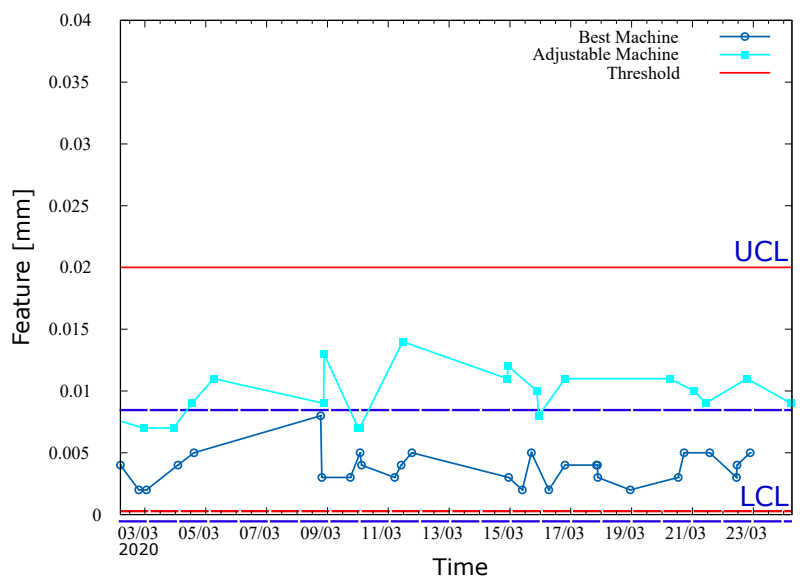

Fig. 3 Example where machine A_$_{-}{ }_{-} 1$ is identified as the best machine and A_3_2 is identified as one of the adjustable machines.

The best machine is then defined, in a first instance, as the machine that, for a feature or set of features, has at least five measurements for the time period currently being looked at, does not have any of those measurements out of threshold and has the smallest Mean deviation. In the same way, the worst machine is a machine with at least five available measurements which has the highest Mean deviation. From here, a set of adjustable machines are defined as those machines that have a higher Mean deviation compared to the best machine but their measurements fall within 3 SDs and within the upper and lower control limits. This does not include those machines that have a lower Mean deviation compared to the best machine but less than five measurements available. Figure 3 shows an example of the best machine found for a feature $F$ as well as one of the identified adjustable machines. The blue doted lines show the 3SD of the machine identified as the best one. As it can be observed, machine A_3_2 is just slightly worse than A_4_1.

The best machine, worst machine and set of adjustable machines as defined previously will be the first indicator of performance of the process for a particular feature or set of features of one or more part types. If all machines apart from the best machine are adjustable, then the process is in-control and can be potentially improved according to the parameters of the best machine. If no best machine is found, is either because there are still not enough measurements to determine the performance or because all machines are showing measurements out of threshold.

Once these indicators are obtained, it is necessary to complement this information by characterising further the machines and so provide a better understanding of what are the key problems that need to be addressed. 
3.3 Pattern analysis for the characterisation of machine performance

The way in which each machine is further characterised is through the identification of different patterns and rules. Each pattern will contribute to the overall characterisation of the machine performance in a positive or negative way. While some patterns may indicate a re-occurring problem, some may indicate an improvement. For example, an increasing/decreasing trend on the measurements approaching the target value would indicate an improvement. Each pattern will have an associated alert, either green, yellow, or red, depending on the nature of the change. Once all machines are checked for patterns and rules, a summary of alerts will be generated. This summary will provide an overall picture of the stability of the process. This will be further discussed in the visualisation section.

\subsubsection{Increasing/Decreasing Trends}

A trend is present if a sequence of measurements exhibits steady upward or downward decline over its whole length. To identify a trend, the Cox-Stuart test is performed. This is a non-parametric sign test for detecting trends in independent, time-ordered data [11]. Although this test is not as powerful as the Mann-Kendall test [39], the computational effort of this test is lower (increases linearly with the sequence size). The main steps to perform the Cox-Stuart test are shown in Algorithm 1.

Once the trends for each feature/machine are determined, an alert associated with each trend will be recorded. A detected trend that moves away from the nominal will raise a red alert, whilst a trend towards the nominal will raise an amber alert.

\subsubsection{Up/Down Shifts}

Detecting multiple change points in a sequence of values can be computationally expensive. A brute force exact approach would take $O\left(Q n^{2}\right)$ calculations for a sequence of values of size $n$, where $\mathrm{Q}$ is the maximum number of change points. The objective is to find the set of change points $\tau$ that minimise the equation:

$\sum_{i=1}^{m+1}\left[C\left(y_{\left(\tau_{i-1}+1\right): \tau_{i}}\right)\right]+\beta f(m)$

where $\mathrm{C}$ is a cost function (typically the negative loglikelihood) for a segment, $m$ is the number of change points $(m+1$ segments) and $\beta f(m)$ if a penalty to avoid over fitting. It is still an open question how to define a

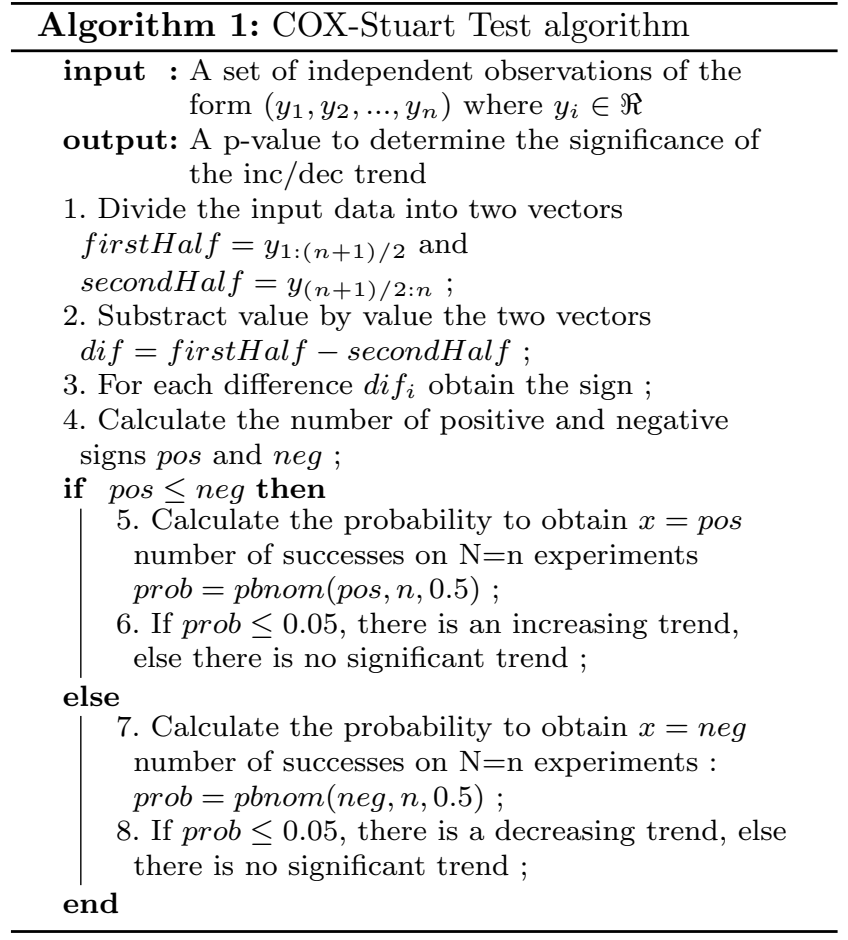

maximum number of change points in a sequence whilst avoiding over fitting.

To check for sudden shifts on the measurements, the Pruned Exact Linear Time (PELT) change point algorithm is used here, with a non-parametric cost function based on the empirical distribution 31. The PELT algorithm is an exact algorithm which is computationally less expensive compared to algorithms such as Binary Segmentation (linear in the best case scenario) by using dynamic programming and pruning. The PELT algorithm is based on the idea that the optimal number of change points of a segment $y_{1: s}(F(s))$ can be expressed in terms of the optimal number of change points for $y_{1: t}$ where $t<s$. This allows to define the optimal number of change points for $y_{1: s}$ recursively in terms of the minimal cost for $y_{1: t}$. To maintain a linear execution time on $n$, this algorithm applies pruning by removing those values of $\tau$ that can never be minima from the minimisation performed at every iteration. The main assumption of the PELT algorithm is that the number of change points increases linearly as the data set grows, in other words, the change points are spread through the data rather than confined to one portion. The steps of this method are presented in Algorithm 2.

Once all change points are found, for each change point detected in a set of measurements if it represents an increase or decrease in $25 \%$ of the tolerance from its previous change point, it is highlighted with a red alert. In addition, for any change point detected in the last 5 


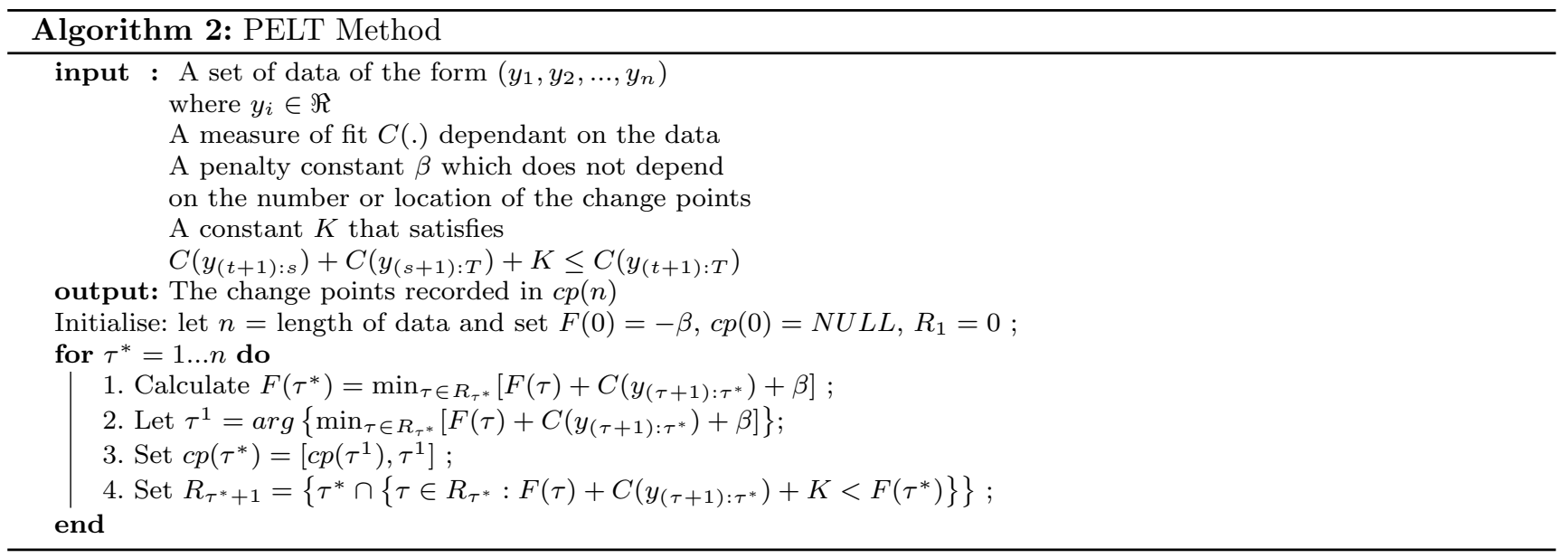

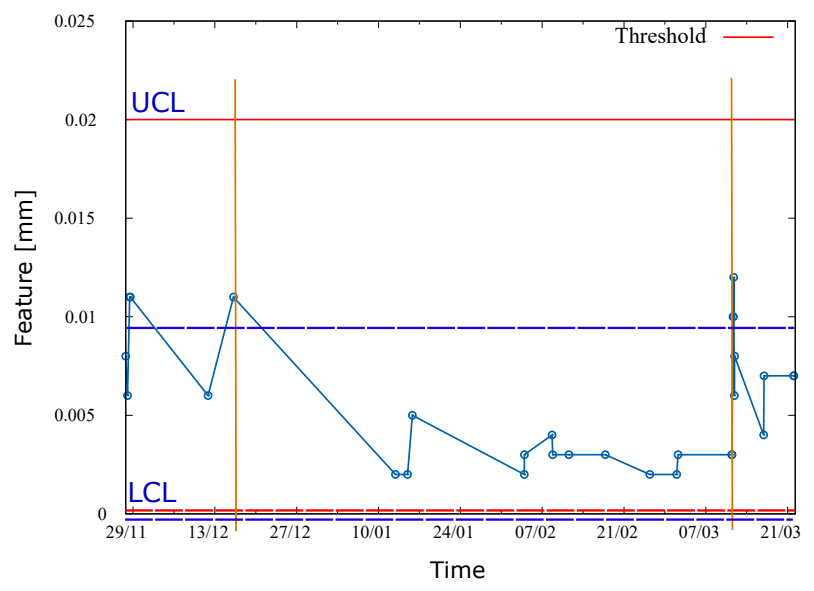

Fig. 4 Detected shifts on a subset of measurements of a sample feature and machine. Each vertical line indicates the point in time where the change is detected. Two moments are identified here, one where there is a tendency towards the target value and one where the measurements start to move away.

observations of a set of measurements, a green alert is raised for those approaching the target value and a red one for those moving away from the target.

\subsubsection{SPC rules}

Standard SPC rules which include increasing/decreasing patterns and saw tooth patterns alternating below and above the target value, have been used to further characterise the machine behaviour. Amber and red alerts are associated with measurements or sets of measurements that meet the criteria of an SPC rule depending on where in time the rule is triggered. Specifically, the following rules have been implemented:

1. One point outside 3 SDs. Any measurement within 3 SDs from the best machine mean would raise an amber alert, while any measurement outside 3 SDs would raise a red alert.
2. Two out of three points in succession out of $3 \mathrm{SD}$ of the best machine mean. Using a rolling window, any 3 consecutive observations of a set of measurements is tested for this rule. A red alert is raised if the rule is triggered by the any of the 3 most recent measurements, or an amber alert is raised if triggered by any of the other observations in the set. This allows to identify the cases where the rule has been triggered recently or if it has been triggered at all.

3. Four out of five points in succession out of 3SD of the best machine mean. Similar to the rule 2 out of 3 , any 4 points in 5 consecutive observations would raise a red alert if triggered on the last three measurements or an amber alert if triggered before.

4. Six points in succession rising or falling. If the last observation is part of an increasing or decreasing trend that is moving away from the nominal, then a red alert will be raised. For any other triggers, an amber alert will be raised.

5. Eight points in succession outside of $1 \mathrm{SD}$. As in previous rules, any triggers in the last 3 measurements will trigger a red alert, while other measurements will trigger an amber alert.

6. Nine points in succession on the same side. Any trigger of this rule will raise a red alert.

7. Fourteen points in succession alternating above and below the target. As with the previous rule any trigger of this rule will raise a red alert.

All the alerts raised by patterns, shifts and SPC rules are used to generate a summary per machine. Results of all machines are then sorted by severity (red alerts having the highest severity and green the least), allowing to identify the machines that have multiple severe alerts Both the summary and results per rule can be visualised through the visualisation tool for further inspection as it will be shown in the next section. 


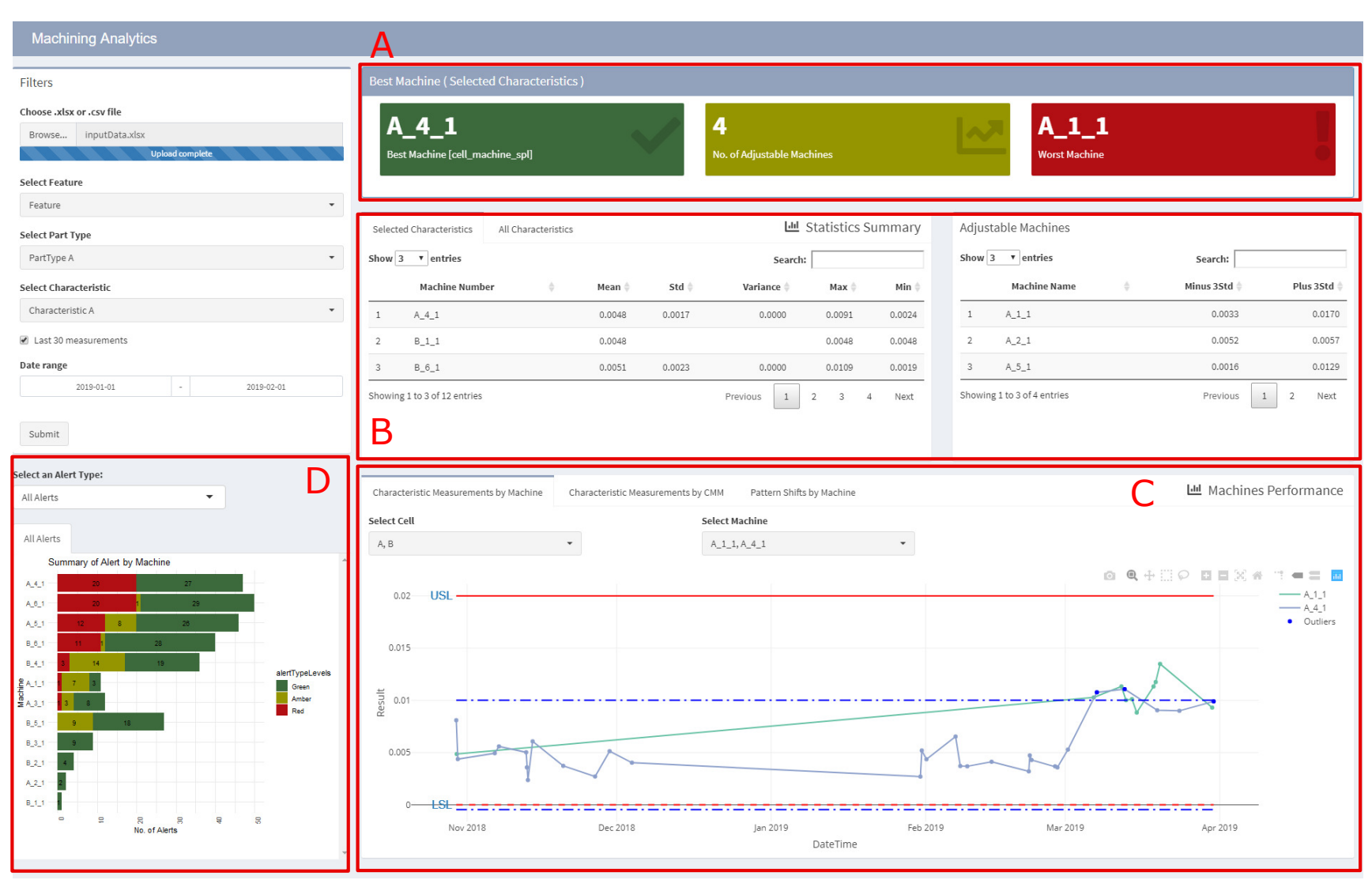

Fig. 5 Visualisation Dashboard consisting of four main visualisations: status panel, statistic tables, alerts summary and control charts.

\subsection{Visualisation tool with $\mathrm{R}$}

Visualising the statistical results and SPC rules is crucial for manufacturing practitioners to understand the general behaviour of the machines in the manufacturing cell and make decisions based on this. The visualisation dashboard that has been implemented (Fig 5 ) has four main visualisation elements which are explained below.

\subsubsection{Status Panel}

As the characterisation of machines for a given feature or set of features is based around the definition of a reference model or best machine, the dashboard provides a quick way for the operator to identify the reference model and related information. This is done through three status boxes at the top of the dashboard (Section A in Figure 5); a green box, which indicates the best machine, a red box which indicates the worst performing machine and an amber box, which indicates the number of adjustable machines (if any).

\subsubsection{Summary Statistics Tables}

To support the results shown in the status panel, the summary statistic tables present the statistical results (mean, SD, variance, max and min) that are computed for each machine and are presented in an ordered manner in section B in Figure 5), left-hand side table. Details of the adjustable machines are presented as well (righ-hand side table).

\subsubsection{Control Chart View}

The control chart view (Section $\mathrm{C}$ in Figure 5 ) allows to visualise the measurements corresponding to a selected feature and time frame for all or sets of machines, highlighting the $3 \mathrm{SD}$ of the best machine (blue dotted line), the outliers (blue dots) and providing the regular elements of a control chart such as the lower and upper control limits (shown in red lines). Data can also be inspected by the CMM machine that performed the measurements, as sometimes there is a consistent error in the measurement machine rather than issues with a particular machine on the manufacturing cell. Finally, it provides a way to visualise change points per machine 


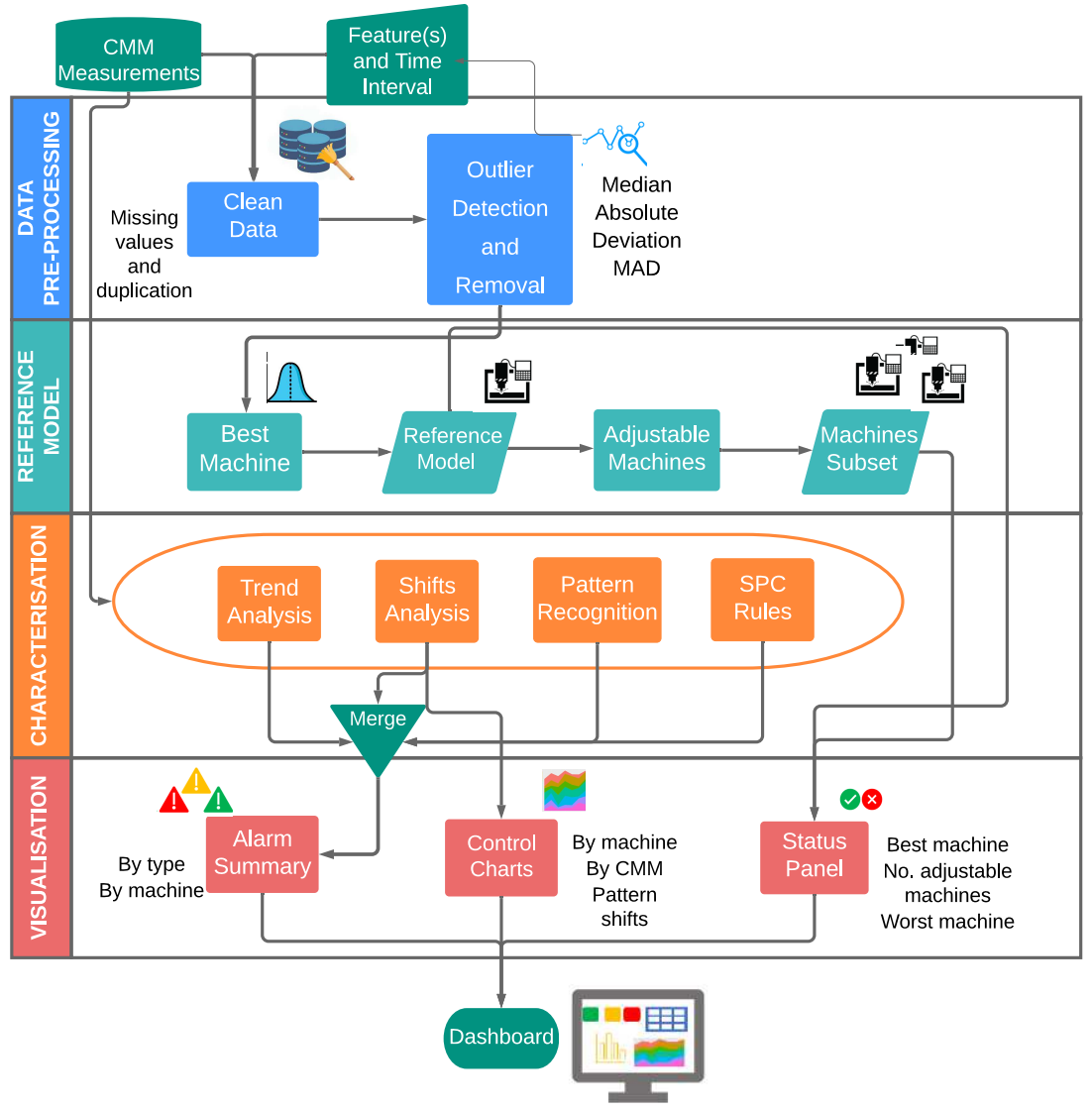

Fig. 6 Methodology used to automatically identify in and out-of-control processes as well as anticipate potential problems occurring at different machines in a flexible manufacturing line.

if the quality practitioner requires further details on the befaviour of a particular machine.

\subsubsection{Alert Summary and details view}

The alert summary (Section D in Figure 5) provides in a first instance a general view of the machines' performance by displaying in order of severity the alerts generated per machine. This allows the operator to identify the machines that are currently more problematic, displaying a large number of red alerts, but also identify which machines could be potentially adjusted as they start showing early signs of a change in the quality of the machined parts. This dashboard feature also allows to filter the summary per rule, which provides further inspection of all the alerts raised by a machine for a particular rule.

All the steps and algorithms of the proposed methodology are sumarised in Figure6. 6 .

\section{Industrial Case Study}

The proposed methodology and visualisation tool was tested in a real flexible manufacturing cell at BMW Plant Hams Hall. The cell configuration is similar to the one shown in Figure 7, where there are between 15 and 20 machines per cell/process, 2 spindles or fixtures per machine and so generally 2 tools per machine. For 8 product types, for example, there can be around 400 measured features, which makes an estimated 96,000 control charts to monitor.

During regular operation of the machines, although appropriate warning limits allow operators to determine the scope of which features need to be reacted to, processes or features which have been affected or shifted but still fall within acceptable limits are often missed. This is directly reflected on the amount of scrap that is produced. Furthermore, the tolerance bands can vary between features and their significance. There are some characteristics which have a range of less than 10 microns and some which can have a range up to $100 \mathrm{mi}-$ crons. 


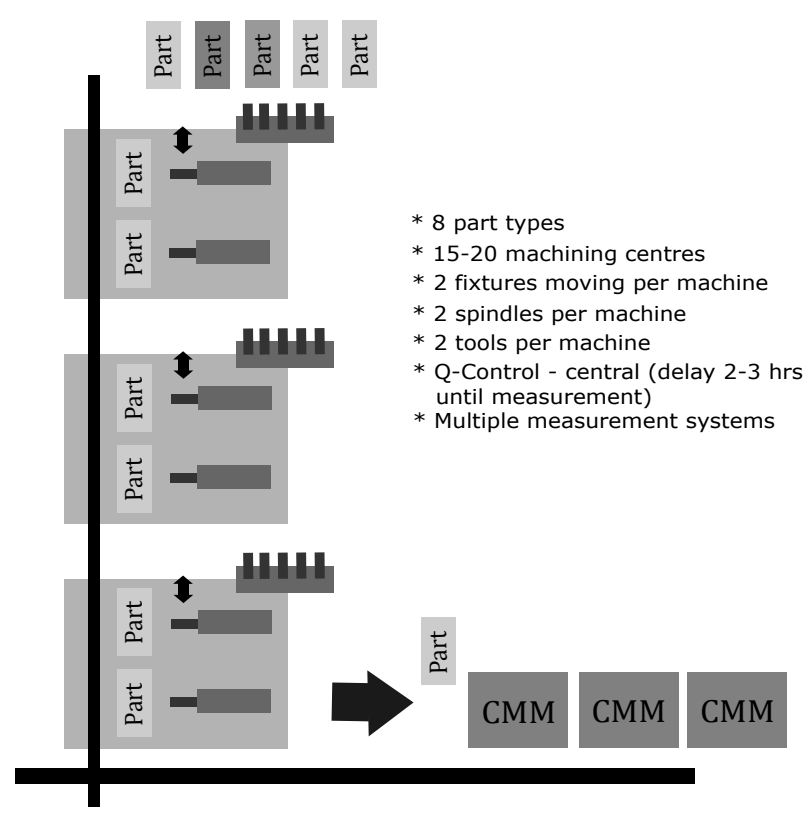

Fig. 7 Typical layout of a Flexible Manufacturing Line at Hams Hall with 15 to 20 machine centres and 2 fixtures and spindles per machine.

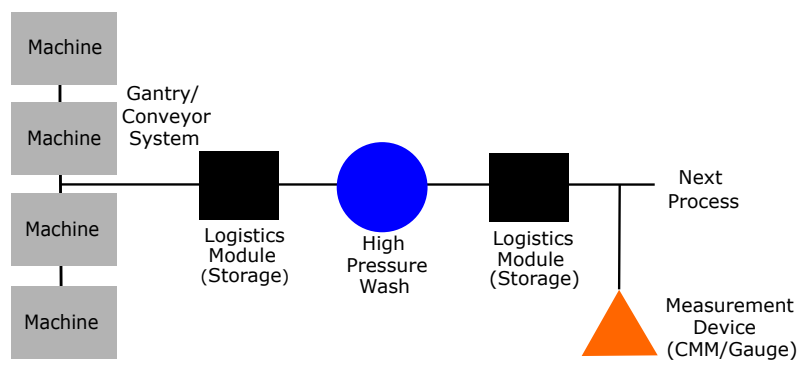

Fig. 8 Selected parts for measurement move through a conveyor system in order to be high-preassure washed before they go through the measurement process.

Additional challenges are imposed by the feature measurement variation. Firstly, measurement duration can take up to 90 minutes for particularly complex processes with 400-500 features on CMMs. This is often why the sampling frequency cannot typically be increased across the whole process. As a result, the lead or 'reference' machine concept is used. The frequency of measure on a single machine within a process group (with multiple machines) is increased and the results of the remaining machines is analysed based on the mean and sparsity of results. Secondly, there is a limited amount of measurement capacity in terms of CMMs because of factors such as the process flow of material through the logistics modules and wash processes. The gauge systems require stringent cleanliness due to the product tolerances (within microns) so they often have to go through high pressure wash systems before measurement (see Fig 8). This all adds to process time and can reduce capacity. Finally, technologies of the mea-

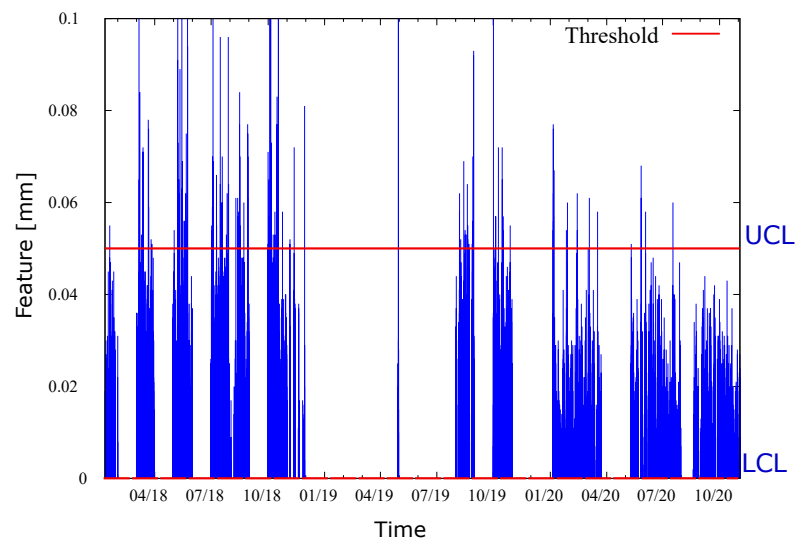

Fig. 9 Measurements of a single feature captured for all monitored machines during the period 2018-2020.

\begin{tabular}{ccc}
\hline Machine Number & Before Tool & After Tool \\
\hline Machine 1 & 0.61 & 2.50 \\
Machine 2 & 0.43 & 1.46 \\
Machine 3 & 0.42 & 1.54 \\
\hline
\end{tabular}

Table 1 Process capability index (cpk) of three machines before using the analyics tool (all measurements taken in 2018) and after using the tool (period of 11 months in 2020).

surement devices also vary, not only with their own resolution and measurement accuracy, but with data accuracy and transfer.

To avoid disruption on the production lines, the tool was deployed progressively, used in a first instance by the quality specialists to monitor a focused range of part types and support process capability reviews carried out and at a later stage by operators to steer daily operations for processes that needed more attention. Machine operators would select a range of features from their operation and use the 'reference model' and the 'adjustable machines' graphic to help evaluate how they could improve the processes. Once standard practice of using the tool was established, the tool was used across an 11 month period (January to November 2020), where continuous machine adjustments were made to help centre the processes. These adjustments were recorded each time to enabled cross shift communication to recognise the best adjustment techniques on the machines.

By being able to identify the key processes to adjust through the analytics tool, the performance of the monitored machines was significantly improved. Figure 9 shows all the measurements taken for a single feature in all machines for a period of two years, from 2018 to 2020. This feature has a unilateral tolerance, where the upper control limit is 0.5 . It can be observed in the figure that CMM measurements start to reflect a more stable and controlled process as measurements out of tolerance are less frequent, especially towards the 

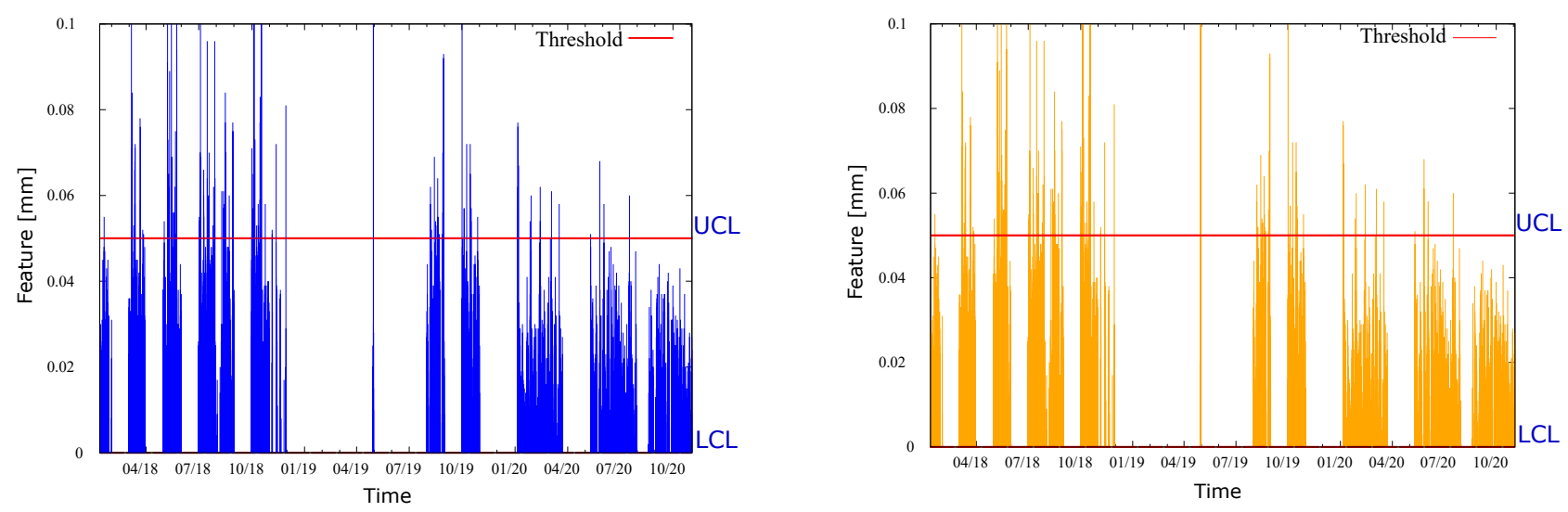

Fig. 10 Measurements of a single feature captured for Machine 1 (left) and Machine 2 (right) during the period 2018-2020
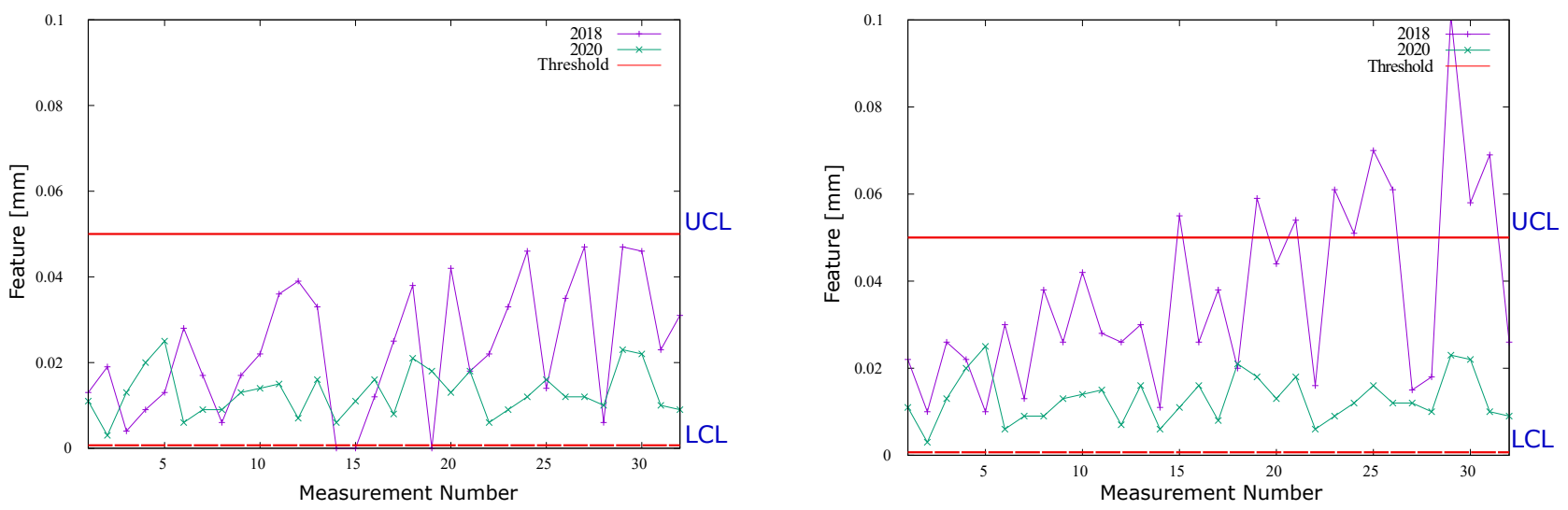

Fig. 11 Thirty two consecutive measurements of a single feature captured for Machine 1 (left) and Machine 2 (right) during the periods 2018 and 2020.

second half of 2020. Inspecting individual machines as shown in Figures 10 and 11, it can be observed that this tendency is true for all the monitored machines. When comparing the process capability index of machines corresponding to measurements taken in 2018 to those in 2020 , it was found that there was a significant improvement across all machines, maintaining a high percentage of produced parts that meet the quality requirements. Initial usage of the tool demonstrated a reduction in $97 \%$ of scrap produced for a focused process and group of features within the 11 month period compared the the previous year. With these initial results, it can be estimated that $80 \%$ of scrap will be reduced from dimensional process faults. Furthermore, with wider use throughout the shop floor, an estimated 500 hours of lost process time can be expected to be gained from reduced validation measurements and wasted process steps.

\section{Conclusion and Future Work}

In this work, a methodology for characterising machine performance through data analytics is presented. The methodology, which has been integrated into a visualisation tool, allows the quality practitioner to draw out the key features to analyse and to identify the ones that have adjustable capabilities within their scope, anticipating this way potential problems. This is crucial, particularly when there is a large number of features to monitor and the level of sensitivity in error is high. Initial usage of the tool in a flexible manufacturing cell at BMW has demonstrated a reduction of $97 \%$ of scrap for the monitored features compared to the previous year. This in the long run will reduce reaction time in following quality control procedures. Furthermore, it will reduce significant scrap costs and ultimately reduce required measurement capacity and enable more output in terms of volume capacity. Further work will include the study of a wider use of the tool throughout the shop floor, analising the usability from the perspective 
of the shop-floor workers, as well as the incorporation of more advanced machine learning algorithms for pattern recognition. Ultimately prescriptive analytics will be explored to provide an intelligent recommendation of parameter adaptation.

\section{Declarations}

\subsection{Ethics Approval}

No ethical approval was needed for this research as no personal data was gathered.

\subsection{Consent to Participate}

All participants in the visualisation tool evaluation are employees of BMW Hams Hall Plant and gave informed affirmative consent to the authors to have their evaluation of the tool included in this paper. No personally identifying information was collected.

\subsection{Consent to Publish and Authors Contributions}

This paper presents original work that is not under consideration in any other journal. All authors approved the manuscript and this submission. The authors contributions were as follows: Dr Giovanna Martinez-Arellano contributed with the draft preparation, methodology and software implementation, Dr ThuBa Nguyen contributed with software implementation, Chris Hinton with the conceptualisation of the study, methodology, data curation and writing and Dr Svetan Ratchev with the conceptualisation of this study.

\subsection{Funding}

This work was funded by BMW Hams Hall Plant, the ESPRC Impact Acceleration Account grant agreement $\mathrm{EP} / \mathrm{R} 511730 / 1$ and the EPSRC Manufacturing on a Shoestring grant agreement EP/R032777/1.

\subsection{Competing Interests}

There are no conflicts of interest in regards to the work presented in this manuscript.
6.6 Availability of data and materials

Data cannot be made publicly available due to a confidentiality agreement with BMW Hams Hall Plant. Due to this agreement, the visualisation tool that has been developmed as part of this work is not available for public use.

\section{References}

1. Amiri A, Allahyari S (2012) Change point estimation methods for control chart postsignal diagnostics: A literature review. Quality and Reliability Engineering International 28(7):673-685, DOI 10.1002/qre.1266, URL https://onlinelibrary.wiley.com/doi/abs/ 10.1002/qre.1266 https://onlinelibrary. wiley.com/doi/pdf/10.1002/qre.1266

2. Arbel A, Seidmann A (1984) Performance evaluation of flexible manufacturing systems. IEEE Transactions on Systems, Man, and Cybernetics SMC-14(4):606-617, DOI 10.1109/TSMC.1984. 6313332

3. Azadeh A, Zeynali J (2008) Design and development of an integrated quality control system. International Journal of Productivity and Quality Management 3(2):183-205, DOI 10.1504/IJPQM.2008. 016564

4. Calzada ME, Scariano SM (2013) The synthetic t and synthetic ewma t charts. Quality Technology \& Quantitative Management 10(1):37-56, DOI 10.1080/16843703.2013.11673307, URL https: //doi.org/10.1080/16843703.2013.11673307, https://doi.org/10.1080/16843703.2013. 11673307

5. Castagliola CGFSea P (2013) The variable sample size $\mathrm{t}$ control chart for monitoring short production runs. International Journal of Advanced Manufacturing Technology pp 1353-1366, DOI $10.1007 / \mathrm{s} 00170-012-4413-8$

6. Castillo ED, Montgomery DC (1994) Short-run statistical process control: Q-chart enhancements and alternative methods. Quality and Reliability Engineering International 10(2):87-97, DOI 10.1002/ qre.4680100203

7. Chen Z, Lu S, Lam S (2007) A hybrid system for spc concurrent pattern recognition. Advanced Engineering Informatics 21(3):303 - 310, DOI https://doi.org/10.1016/j.aei.2007. 03.002, URL http://www.sciencedirect.com/ science/article/pii/S1474034607000225, applications Eligible for Data Mining 
8. Chung-Hsien Kuo, Han-Pang Huang (2000) Failure modeling and process monitoring for flexible manufacturing systems using colored timed petri nets. IEEE Transactions on Robotics and Automation 16(3):301-312, DOI 10.1109/70.850648

9. Cordero R (1997) Changing human resources to make flexible manufacturing systems (fms) successful. The Journal of High Technology Management Research 8(2):263 - 275, DOI https://doi.org/10.1016/S1047-8310(97)90006-7, URL http://www.sciencedirect.com/science/ article/pii/S1047831097900067

10. Costa AFB, Castagliola P (2011) Effect of measurement error and autocorrelation on the $\mathrm{x}^{-}$chart. Journal of Applied Statistics 38(4):661-673, DOI 10.1080/02664760903563627, URL https://doi. org/10.1080/02664760903563627, https://doi. org/10.1080/02664760903563627

11. COX DR, STUART A (1955) SOME QUICK SIGN TESTS FOR TREND IN LOCATION AND DISPERSION. Biometrika 42(1-2):80-95, DOI 10. 1093/biomet/42.1-2.80, URL https://doi.org/ 10.1093/biomet/42.1-2.80

12. Diez-Olivan A, Ser] JD, Galar D, Sierra B (2019) Data fusion and machine learning for industrial prognosis: Trends and perspectives towards industry 4.0. Information Fusion 50:92 - 111, DOI https://doi.org/10.1016/j.inffus.2018.10.005, URL http://www.sciencedirect.com/science/ article/pii/S1566253518304706

13. Ding Y, Zeng L, Zhou S (2006) Phase i analysis for monitoring nonlinear profiles in manufacturing processes. Journal of Quality Technology 38(3):199-216, DOI 10.1080/00224065. 2006.11918610, URL https://doi.org/10.1080/ 00224065.2006.11918610, https://doi.org/10. 1080/00224065.2006.11918610

14. Ge Z, Song Z, Ding SX, Huang B (2017) Data mining and analytics in the process industry: The role of machine learning. IEEE Access 5:20590-20616, DOI 10.1109/ACCESS.2017.2756872

15. Gu K, Jia X, You H, Zhang S (2014) A tchart for monitoring multi-variety and small batch production run. Quality and Reliability Engineering International 30(2):287-299, DOI 10.1002/qre.1496, URL https://onlinelibrary. wiley.com/doi/abs/10.1002/qre.1496, https://onlinelibrary.wiley.com/doi/pdf/ 10.1002/qre.1496

16. Guerra L, Sousa SD, Nunes EP (2016) Statistical process control automation in the final inspection process: An industrial case study. In: 2016 IEEE International Conference on Industrial Engineering and Engineering Management (IEEM), pp 876-880, DOI 10.1109/IEEM.2016.7798002

17. Guh RS (2003) Integrating artificial intelligence into on-line statistical process control. Quality and Reliability Engineering International 19(1):1-20, DOI 10.1002/qre.510, URL https://onlinelibrary.wiley.com/doi/abs/ 10.1002/qre.510 https://onlinelibrary. wiley.com/doi/pdf/10.1002/qre.510

18. Hawkins DM, Deng Q (2010) A nonparametric change-point control chart. Journal of Quality Technology 42(2):165-173, DOI 10.1080/00224065. 2010.11917814, URL https://doi.org/10.1080/ 00224065.2010.11917814, https://doi.org/10. 1080/00224065.2010.11917814

19. Hawkins DM, Qiu P, Kang CW (2003) The changepoint model for statistical process control. Journal of Quality Technology 35(4):355-366, DOI 10.1080/00224065.2003.11980233, URL https: //doi.org/10.1080/00224065.2003.11980233, https://doi.org/10.1080/00224065.2003. 11980233

20. He F, Jiang W, Shu L (2008) Improved self-starting control charts for short runs. Quality Technology \& Quantitative Management 5(3):289-308, DOI 10.1080/16843703.2008.11673402, URL https: //doi.org/10.1080/16843703.2008.11673402, https://doi.org/10.1080/16843703.2008. 11673402

21. He QP, Wang J (2018) Statistical process monitoring as a big data analytics tool for smart manufacturing. Journal of Process Control 67:35-43, DOI https://doi.org/10.1016/j.jprocont.2017.06.012, URL https://www.sciencedirect.com/ science/article/pii/S0959152417301257.

big Data: Data Science for Process Control and Operations

22. He S, He Z, Wang G (2013) Online monitoring and fault identification of mean shifts in bivariate processes using decision tree learning techniques. Journal of Intelligent Manufacturing 24:25-34, DOI 10.1007/s10845-011-0533-5

23. Herrmann C, Schmidt C, Kurle D, et al. (2014) Sustainability in manufacturing and factories of the future. International Journal of Precision Engineering and Manufacturing-Green Technology pp 283-292, DOI 10.1007/s40684-014-0034-z

24. Hillier FS (1969) $\overline{\mathrm{X}}$ - and r-chart control limits based on a small number of subgroups. Journal of Quality Technology 1(1):17-26, DOI 10.1080/00224065. 1969.11980343, URL https://doi.org/10.1080/ 00224065.1969.11980343, https://doi.org/10. 1080/00224065.1969.11980343 
25. Huang CT, Yeh TM, Lin WT, Lee BT (2009) A fuzzy ahp-based performance evaluation model for implementing $\mathrm{spc}$ in the taiwanese lcd industry. International Journal of Production Research 47(18):51635183, DOI $10.1080 / 00207540801935608$, URL https://doi.org/10.1080/00207540801935608, https://doi.org/10.1080/00207540801935608

26. ISO 9002:2015 (2015) Quality management standards. Standard, International Organisation for Standardisation

27. Jiang W, Tsui KL, Woodall WH (2000) A new spc monitoring method: The arma chart. Technometrics 42(4):399-410, DOI 10.1080/00401706. 2000.10485713, URL https://www.tandfonline. $\mathrm{com} / \mathrm{doi} / \mathrm{abs} / 10.1080 / 00401706.2000 .10485713$

28. Jones-Farmer LA, Woodall WH, Steiner SH, Champ CW (2014) An overview of phase i analysis for process improvement and monitoring. Journal of Quality Technology 46(3):265-280, DOI 10.1080/00224065.2014.11917969, URL https: //doi.org/10.1080/00224065.2014.11917969, https://doi.org/10.1080/00224065.2014. 11917969

29. Kapoor Y, Meyer R, Meyer B, et al. (2020) Flexible manufacturing: The future state of drug product development and commercialization in the pharmaceutical industry. Journal of Pharmaceutical Innovation DOI https://doi.org/10.1007/ s12247-019-09426-z

30. Khoo MBC (2003) Design of runs rules schemes. Quality Engineering 16(1):2743, DOI 10.1081/QEN-120020769, URL https://doi.org/10.1081/QEN-120020769, https://doi.org/10.1081/QEN-120020769

31. Killick R, Fearnhead P, Eckley IA (2012) Optimal detection of changepoints with a linear computational cost. Journal of the American Statistical Association 107(500):1590-1598, DOI 10. 1080/01621459.2012.737745, URL https://doi. org/10.1080/01621459.2012.737745, https:// doi.org/10.1080/01621459.2012.737745

32. Koh CK, Chin JF, Kamaruddin S (2019) Modified short-run statistical process cotrol for test and measurement process. The International Journal of Advanced Manufacturing Technology 100:1531-1548, DOI 10.1007/s00170-018-2776-1

33. Korzenowski A, Vidor G, Vaccaro G, Ten Caten C (2015) Control charts for flexible and multivariety production systems. Comput Ind Eng 88(C):284-292, DOI $10.1016 /$ j.cie.2015.07.016, URL https://doi.org/10.1016/j.cie.2015. 07.016
34. L Zhang GC, Castagliola P (2009) On t and ewma t charts for monitoring changes in the process mean. Quality and Reliability Engineering International (8):933-945, DOI 10.1002/qre.1012

35. Leys C, Ley C, Klein O, Bernard P, Licata L (2013) Detecting outliers: Do not use standard deviation around the mean, use absolute deviation around the median. Journal of Experimental Social Psychology 49(4):764 - 766, DOI https://doi.org/10.1016/j.jesp.2013. 03.013, URL http://www.sciencedirect.com/ science/article/pii/S0022103113000668

36. Liao Y, Deschamps F, de Freitas Rocha Loures E, Ramos LFP (2017) Past, present and future of industry 4.0 - a systematic literature review and research agenda proposal. International Journal of Production Research 55(12):3609-3629, DOI 10. 1080/00207543.2017.1308576, URL https://doi. org/10.1080/00207543.2017.1308576, https:// doi.org/10.1080/00207543.2017.1308576

37. Mahmood K, Karaulova T, Otto T, Shevtshenko E (2017) Performance analysis of a flexible manufacturing system (fms). Procedia CIRP 63:424 - 429, DOI https://doi.org/10.1016/j.procir.2017.03.123, URL http://www.sciencedirect.com/science/ article/pii/S2212827117302706, manufacturing Systems 4.0 - Proceedings of the 50th CIRP Conference on Manufacturing Systems

38. Marques PA, Cardeira C, Paranhos P, Ribeiro S, Gouveia H (2015) Selection of the most suitable statistical process control approach for short production runs: A decision-model. International Journal of Information and Education Technology pp 303310, DOI 10.7763/IJIET.2015.V5.521

39. Militino AF, Moradi M, Ugarte MD (2020) On the performances of trend and change-point detection methods for remote sensing data. Remote Sensing 12(6), DOI 10.3390/rs12061008, URL https: //www.mdpi.com/2072-4292/12/6/1008

40. Miller J (1991) Short report: Reaction time analysis with outlier exclusion: Bias varies with sample size. The Quarterly Journal of Experimental Psychology Section A 43(4):907-912, DOI 10.1080/ 14640749108400962, URL https://doi.org/10. 1080/14640749108400962, https://doi.org/10. $1080 / 14640749108400962$

41. Montgomery DC (2013) Introduction to Statistical Quality Control, seventh edn. Wiley

42. Moyne J, Iskandar J (2017) Big data analytics for smart manufacturing: Case studies in semiconductor manufacturing. Processes 5(3):39, DOI $10.3390 /$ pr5030039 
43. Perry MB, Jr JJP (2006) Estimation of the change point of a normal process mean with a linear trend disturbance in spc. Quality Technology \& Quantitative Management 3(3):325-334, DOI 10.1080/16843703.2006.11673118, URL https: //doi.org/10.1080/16843703.2006.11673118, https://doi.org/10.1080/16843703.2006. 11673118

44. Quesenberry CP (1991) Spc q charts for start-up processes and short or long runs. Journal of Quality Technology 23(3):213-224, DOI 10.1080/00224065. 1991.11979327, URL https://doi.org/10.1080/ 00224065.1991.11979327, https://doi.org/10. 1080/00224065.1991.11979327

45. Reis MS, Gins G (2017) Industrial process monitoring in the big data/industry 4.0 era: from detection, to diagnosis, to prognosis

46. Rychener L, Montet F, Hennebert J (2020) Architecture proposal for machine learning based industrial process monitoring. Procedia Computer Science 170:648 - 655, DOI https://doi.org/10.1016/j.procs.2020.03.137, URL http://www.sciencedirect.com/science/ article/pii/S1877050920305925 the 11th International Conference on Ambient Systems, Networks and Technologies (ANT) / The 3rd International Conference on Emerging Data and Industry 4.0 (EDI40) / Affiliated Workshops

47. Santos MY, e Sá] JO, Andrade C, Lima] FV, Costa E, Costa C, Martinho B, Galvão J (2017) A big data system supporting bosch braga industry 4.0 strategy. International Journal of Information Management 37(6):750 760, DOI https://doi.org/10.1016/j.ijinfomgt.2017. 07.012, URL http://www.sciencedirect.com/ science/article/pii/S0268401217306023

48. Stojanovic L, Dinic M, Stojanovic N, Stojadinovic A (2016) Big-data-driven anomaly detection in industry (4.0): An approach and a case study. In: 2016 IEEE International Conference on Big Data (Big Data), pp 1647-1652, DOI 10.1109/BigData. 2016.7840777

49. Stojanovic N, Dinic M, Stojanovic L (2015) Big data process analytics for continuous process improvement in manufacturing. In: Proceedings of the 2015 IEEE International Conference on Big Data (Big Data), IEEE Computer Society, USA, BIG DATA '15, p 1398-1407, DOI 10.1109/BigData. 2015.7363900, URL https://doi.org/10.1109/ BigData.2015.7363900

50. Tao Zan HwMW Zhihao Liu, Gao X (2020) Control chart pattern recognition using the convolutional neural network. Journal of Intelligent Manufactur- ing 31:703-716, DOI 10.1007/s10845-019-01473-0

51. Toni AD, Tonchia S (1998) Manufacturing flexibility: A literature review. International Journal of Production Research 36(6):1587 1617, DOI 10.1080/002075498193183, URL https : //doi.org/10.1080/002075498193183, https:// doi.org/10.1080/002075498193183

52. Wang KA J, Hannam R (1998) Pattern recognition for statistical process control charts. International Journal of Advanced Manufacturing Tehcnology 14:99-109, DOI 10.1007/BF01322218

53. Wu Z, Shamsuzzaman M, Pan ES (2004) Optimization design of control charts based on taguchi's loss function and random process shifts. International Journal of Production Research 42(2):379-390, DOI 10.1081/ 00207540310001614169, URL https://doi. org/10.1081/00207540310001614169, https: //doi.org/10.1081/00207540310001614169

54. Wu Z, Jiao J, Yang M, Liu Y, Wang Z (2009) An enhanced adaptive cusum control chart. IIE Transactions 41(7):642-653, DOI 10.1080/ 07408170802712582, URL https://doi.org/10. 1080/07408170802712582, https://doi.org/10. 1080/07408170802712582

55. Yasui S, Noguchi H, Ojima Y (2012) A robust detection procedure for multiple change points of linear trends. pp 197-206, DOI 10. 1007/978-3-7908-2846-7-14, 2010 10th International Workshop on Intelligent Statistical Quality Control ; Conference date: 18-08-2010 Through 2008-2010

56. Yu J, Xi L (2009) A neural network ensemblebased model for on-line monitoring and diagnosis of out-of-control signals in multivariate manufacturing processes. Expert Systems with Applications 36(1):909 - 921, DOI https://doi.org/10.1016/j.eswa.2007.10.003, URL http://www.sciencedirect.com/science/ article/pii/S0957417407004939

57. Zamba K, Hawkins D (2006) A multivariate change-point model for statistical process control. Technometrics 48(4):539-549, DOI 10.1198/ 004017006000000291

58. Zan T, Liu Z, Su Z, Wang M, Gao X, Chen D (2020) Statistical process control with intelligence based on the deep learning model. Applied Sciences 10(1), DOI 10.3390/app10010308, URL https://www .mdpi.com/2076-3417/10/1/308

59. Zantek PF (2005) Run-length distributions of qchart schemes. IIE Transactions 37(11):1037-1045, DOI 10.1080/07408170500232297, URL https:// doi.org/10.1080/07408170500232297, https:// 
doi.org/10.1080/07408170500232297

60. Zhao F, He Z, Wu D (2008) Quality assurance of mass customization: A state-of-the-art review. In: 2008 IEEE Symposium on Advanced Management of Information for Globalized Enterprises (AMIGE), pp 1-3, DOI 10.1109/AMIGE.2008. ECP.75

61. Zhou C, Zou C, Zhang Y, Wang Z (2009) Nonparametric control chart based on change-point model. Statistical Papers 50:13-28, DOI 10.1007/ s00362-007-0054-7

62. Zhou JPWX X (2018) Recognition of control chart patterns using fuzzy SVM with a hybrid kernel function. Journal of Intelligent Manufacturing 29:51-67, DOI 10.1007/s10845-015-1089-6 


\section{Figures}

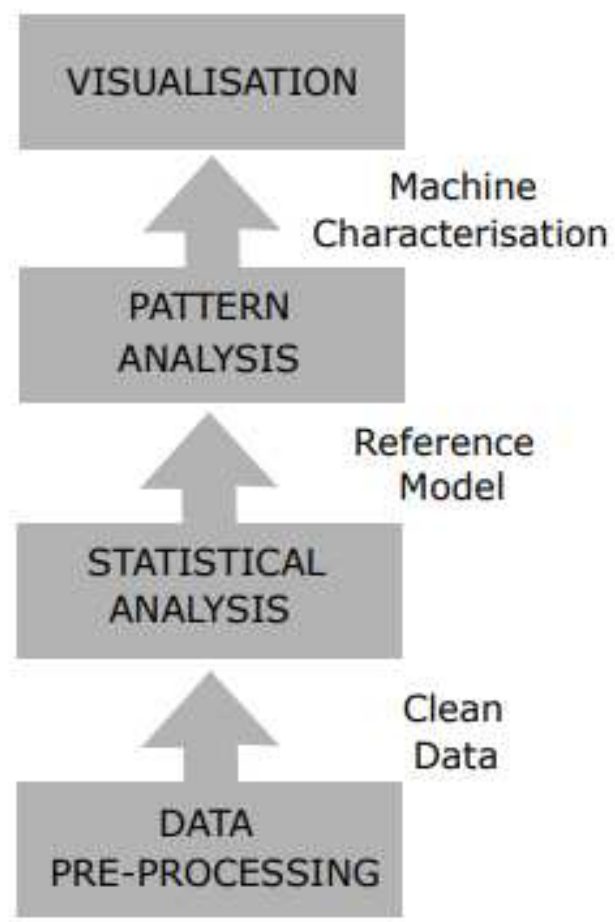

\section{Figure 1}

Methodology used to automatically identify incontrol, out-of-control and adjustable processes in a flexible manufacturing line.

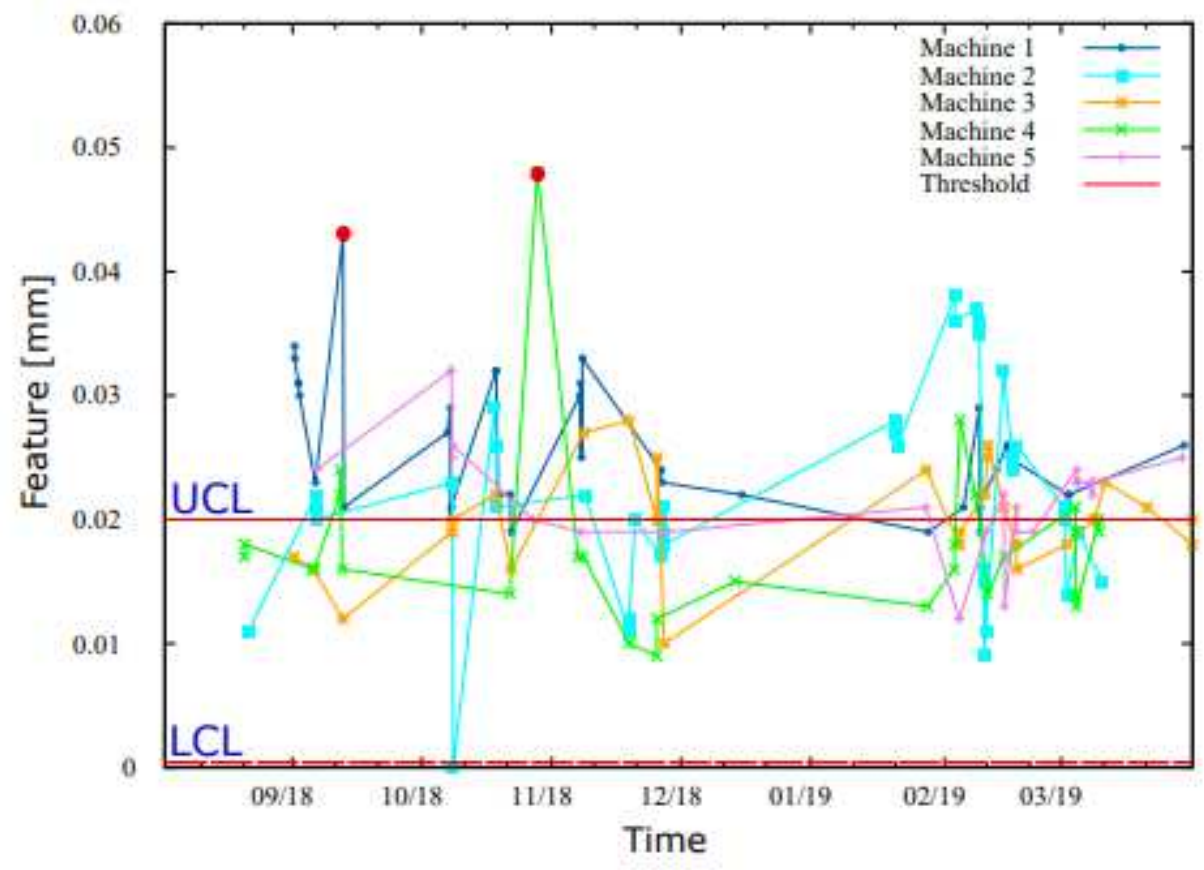

Figure 2 
Outliers (red dots) identified on CMM measurements of a particular feature F corresponding to 5 machines. Although the algorithm picks up an outlier in machine $A 71$, this measurement is not considered an outlier in this particular case as the target value for this feature is zero.

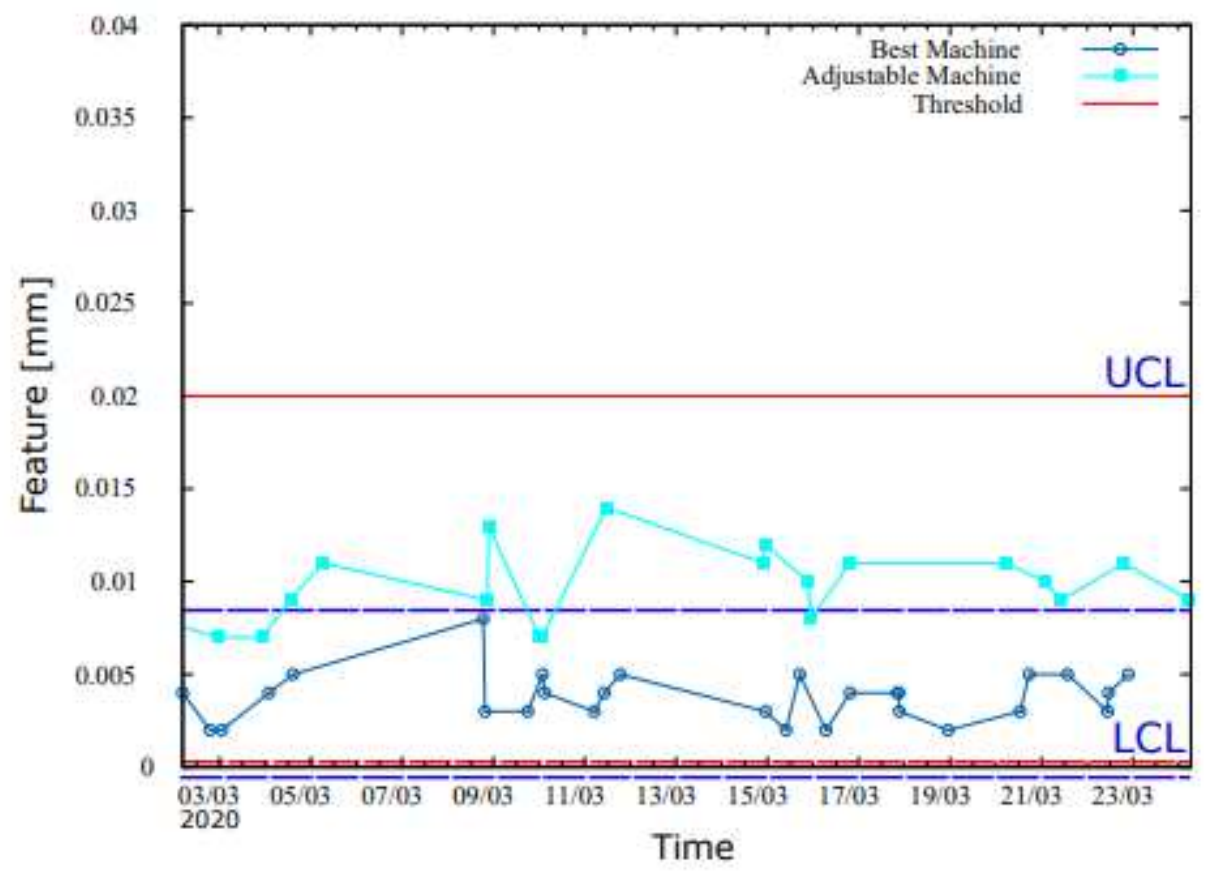

\section{Figure 3}

Example where machine A 41 is identified as the best machine and A 32 is identified as one of the adjustable machines.

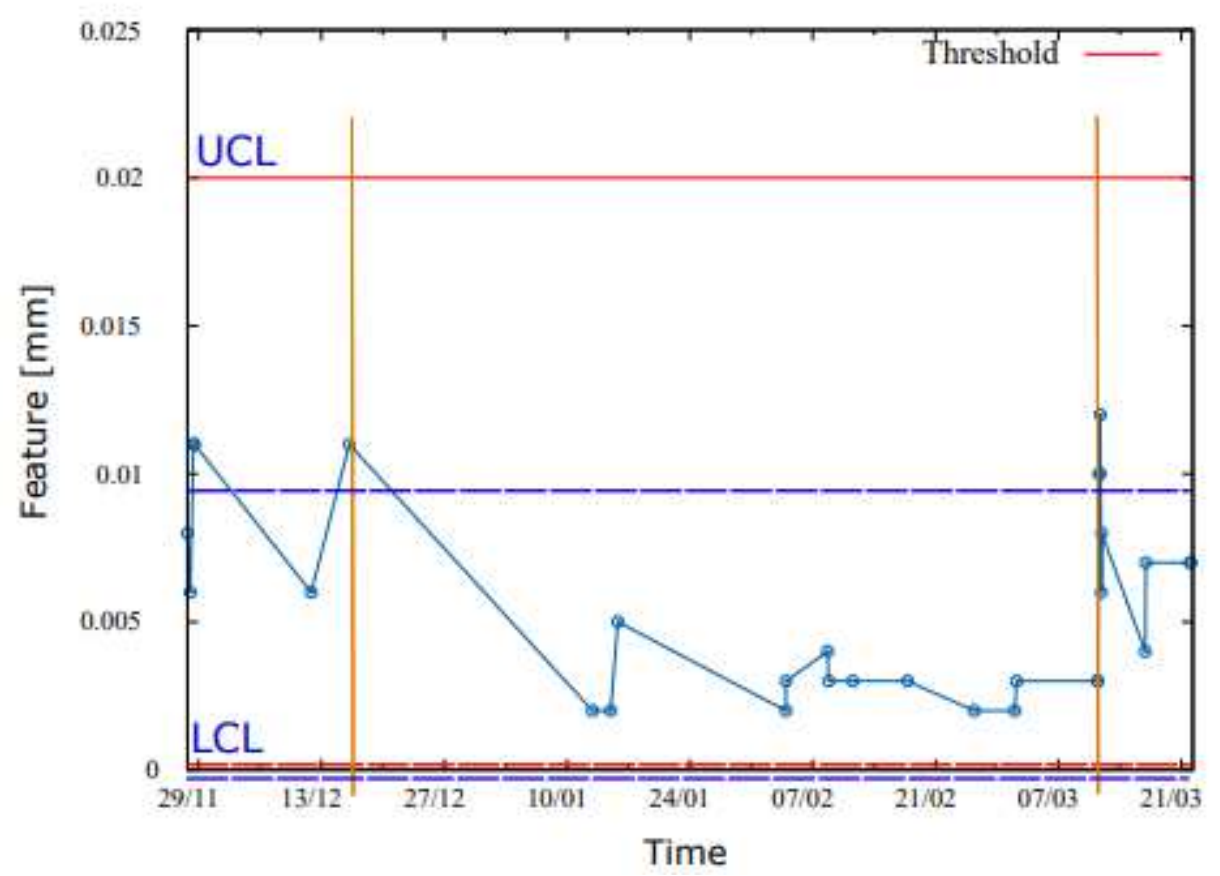

Figure 4 
Detected shifts on a subset of measurements of a sample feature and machine. Each vertical line indicates the point in time where the change is detected. Two moments are identified here, one where there is a tendency towards the target value and one where the measurements start to move away.

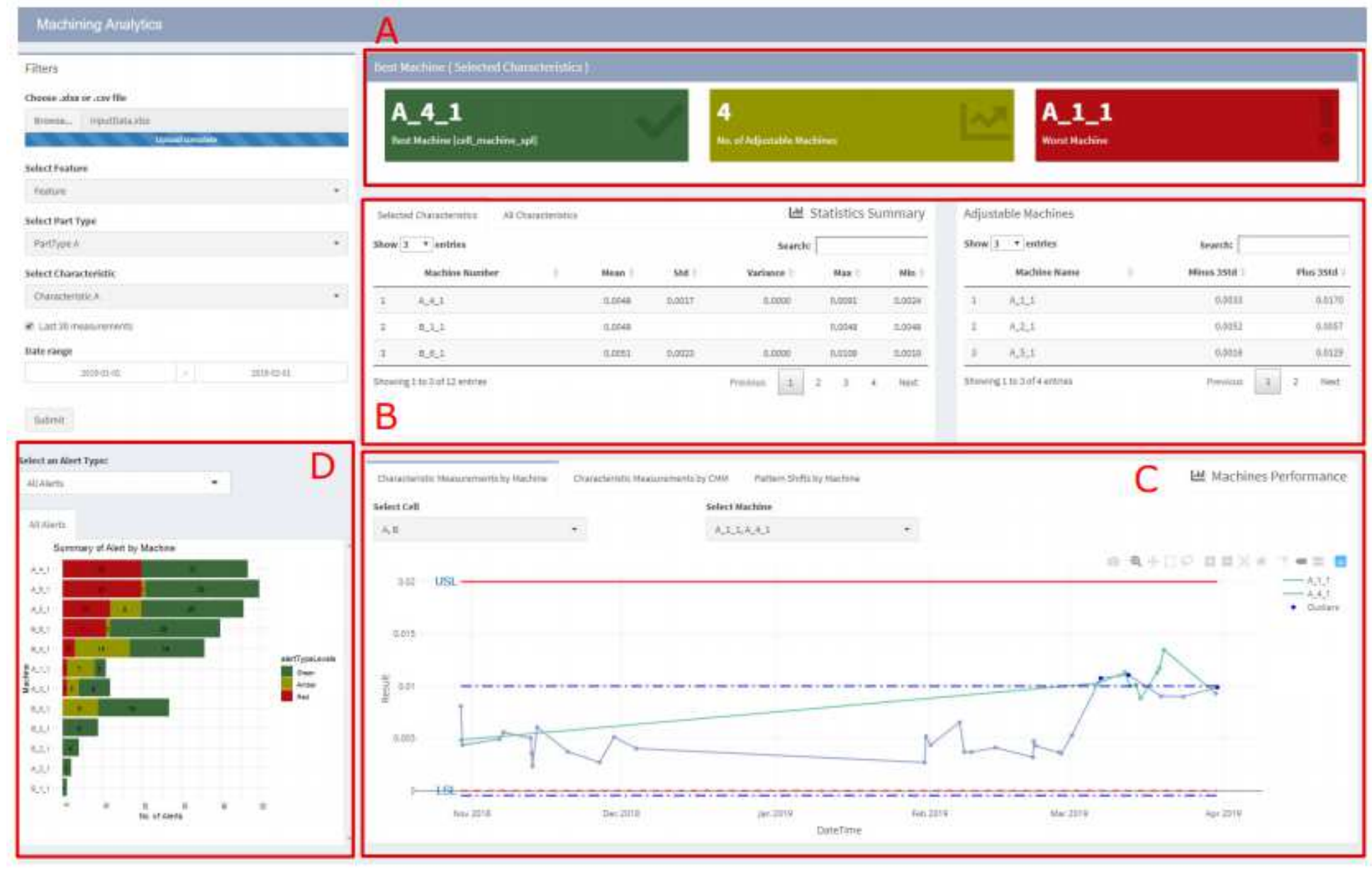

\section{Figure 5}

Visualisation Dashboard consisting of four main visualisations: status panel, statistic tables, alerts summary and control charts. 


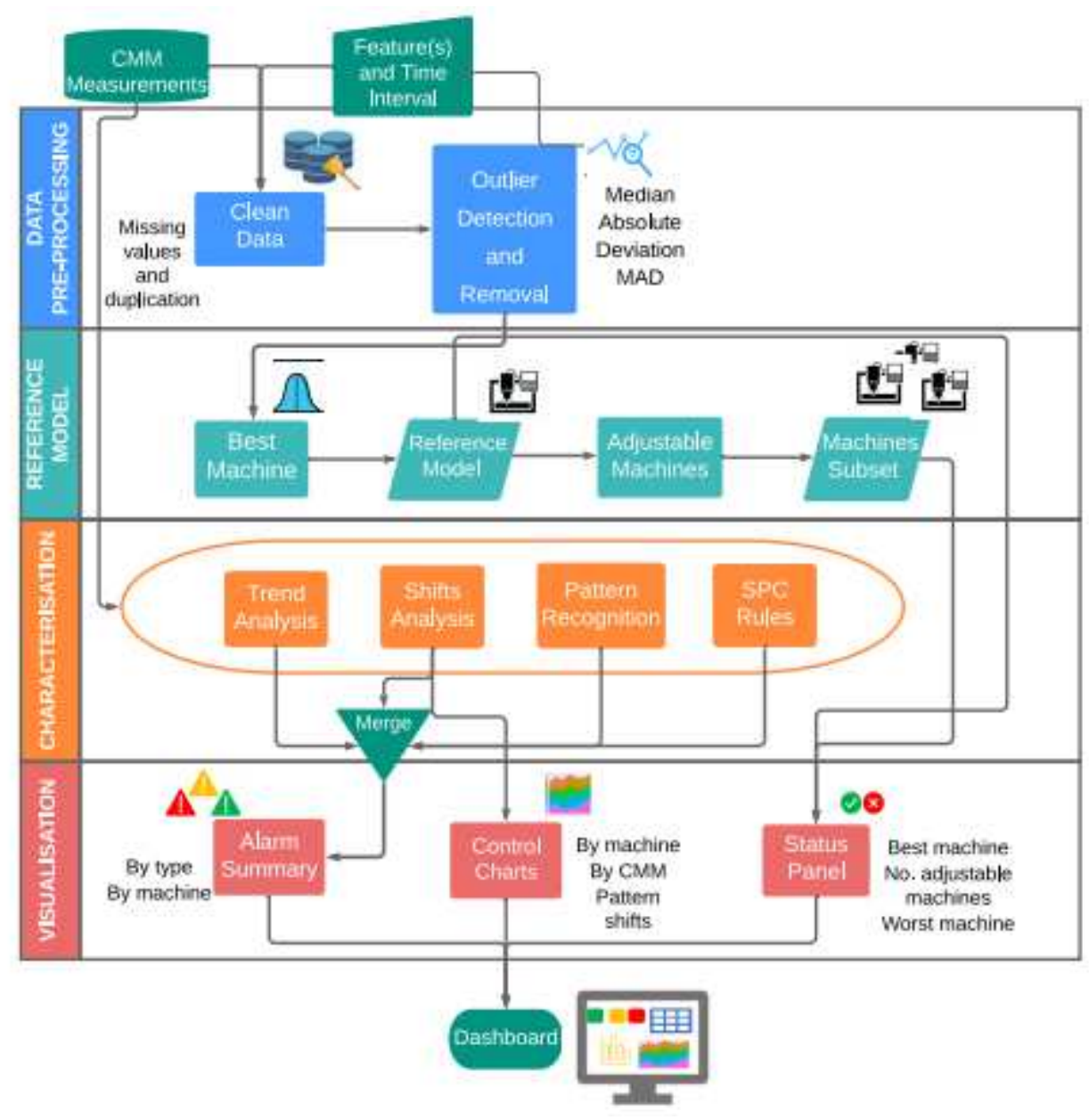

Figure 6

Methodology used to automatically identify in and out-of-control processes as well as anticipate potential problems occurring at different machines in a flexible manufacturing line. 


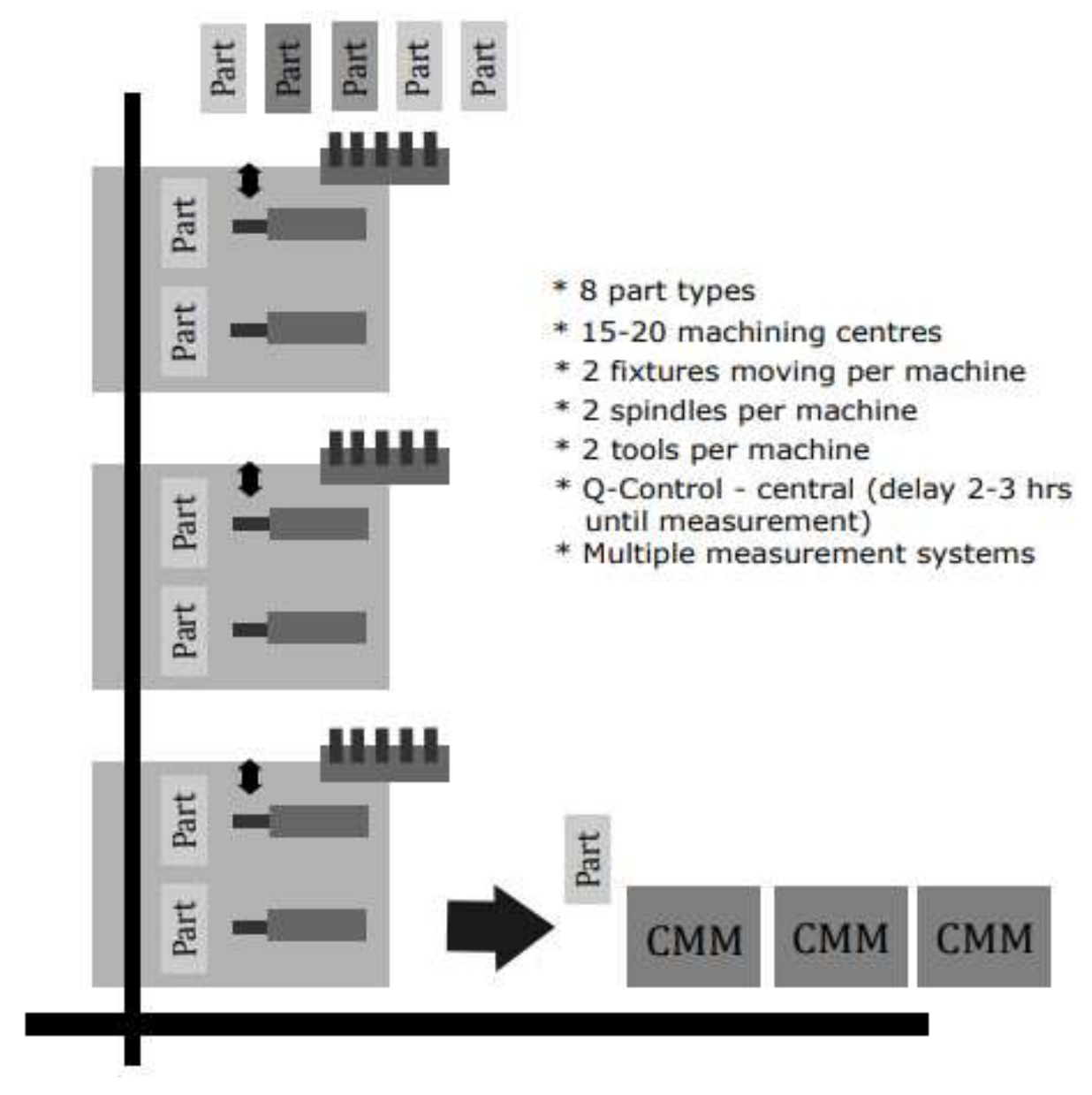

Figure 7

Typical layout of a Flexible Manufacturing Line at Hams Hall with 15 to 20 machine centres and 2 fixtures and spindles per machine.

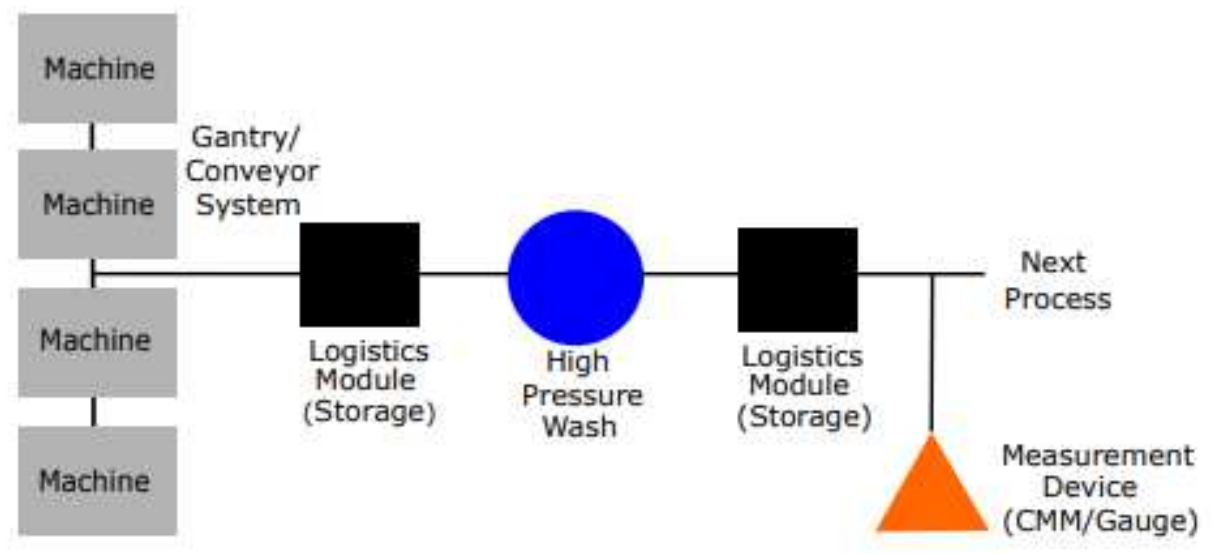

\section{Figure 8}

Selected parts for measurement move through a conveyor system in order to be high-preassure washed before they go through the measurement process. 


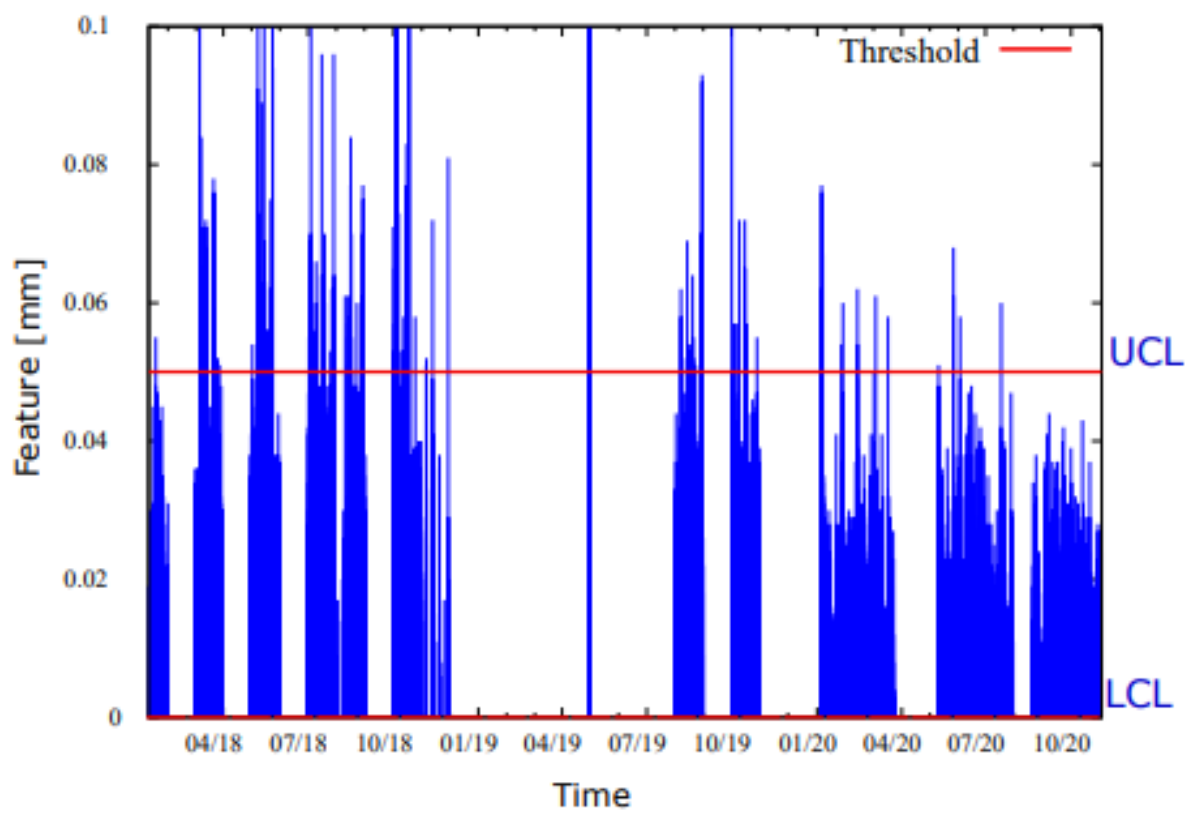

Figure 9

Measurements of a single feature captured for all monitored machines during the period 2018-2020.
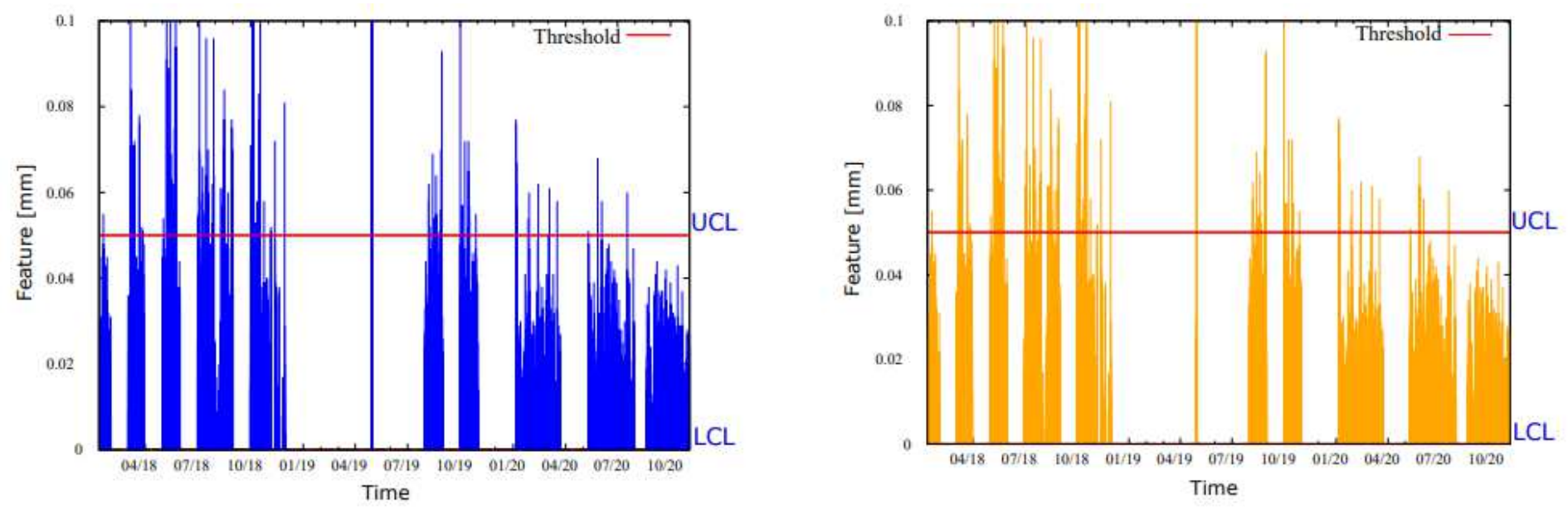

Figure 10

Measurements of a single feature captured for Machine 1 (left) and Machine 2 (right) during the period 2018-2020 

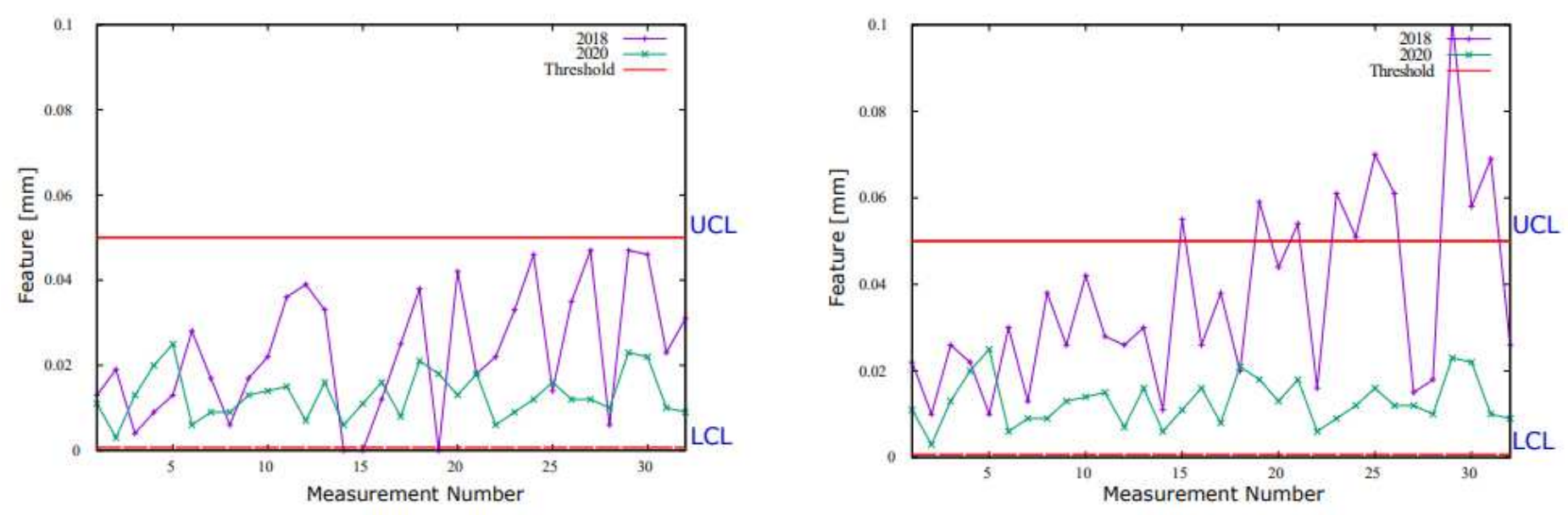

Figure 11

Thirty two consecutive measurements of a single feature captured for Machine 1 (left) and Machine 2 (right) during the periods 2018 and 2020. 